\title{
Quantitative Insights into Energy Contribution of Weak Intermolecular Interactions in Angular Furanocoumarin Derivatives
}

\author{
AHSAN ELAHI and RAJNI KANT* \\ Department of Physics \& Electronics, University of Jammu, Jammu Tawi -180 006, India \\ rkant.ju@gmail.com
}

Received 31 December 2014 / Accepted 21 January 2015

\begin{abstract}
Crystal packing of seven derivatives of angular furanocoumarin derivatives obtained from CSD search is presented in the form of systematic analysis of interaction energies between neighbouring molecular pairs in the crystal rather than in terms of interaction between atoms in neighbouring molecules. Lattice energy of all the compounds and intermolecular energies of neighbouring molecular pairs partitioned into coulombic, polarization, dispersion and repulsion contributions are calculated by PIXEL-semiclassical density sums (SCDS) method. It has been found that aromatic ring stacking $(\pi \ldots \pi)$ contribute more to the cohesive energy of the crystals. Weak intermolecular $\mathrm{C}-\mathrm{H} \ldots \mathrm{O}$ and $\mathrm{C}-\mathrm{H} \ldots \pi$ hydrogen bonds also make significant contributions towards the stabilization of the structure.
\end{abstract}

Keywords: Furanocoumarin, PIXEL, Hydrogen bonding, Intermolecular interactions, Lattice energy

\section{Introduction}

Furanocoumarins are an important class of tricyclic aromatic compounds consisting of a fused structure of coumarin and furan nucleus. Among furanocoumarin derivatives, psoralen and xanthotoxin are most abundant linear furanocoumarin where as the angular type is mostly represented by angelicin and sphondin. Furanocoumarin derivatives are of interest because of their high photobiological activity ${ }^{1-3}$. Both linear and angular furanocoumarins exhibit interesting pharmacological activity, but angular structure reduces the undesirable side effects ${ }^{4}$ such as genotoxicity ${ }^{5}$ and skin cancer $^{6}$ as observed in case of linear furanocoumarin. Therefore, angular furanocoumarins have been paid considerable attention. The occurrence of different kinds of intermolecular interactions in crystal structures will result in different chemical and biological activities which is quite beneficial to pharmaceutical industries ${ }^{7}$. Strong intermolecular interactions such as $\mathrm{N}-\mathrm{H} \ldots \mathrm{O} / \mathrm{N}$ and $\mathrm{O}-\mathrm{H} . . . \mathrm{O} / \mathrm{N}$ are well understood and are found to play a vital role in crystal packing. ${ }^{8}$ In the last few decades, the main focus is to exploit the role of weak intermolecular interactions such as $\mathrm{C}-\mathrm{H} \cdots \mathrm{O} / \mathrm{N}^{9,10}, \mathrm{C}-\mathrm{H} \cdots \mathrm{X}$ (halogen atom $)^{11,12}, \mathrm{C}-\mathrm{H} \cdots \pi,{ }^{13} \pi \cdots \pi{ }^{14}$ and $\mathrm{lp} \cdots \pi^{15}$ interactions present in the crystal structure. Quantitative evaluation of these interactions is required to have 
better understanding of the contribution of these interactions towards crystal packing. In this regard, CSD search has been carried out and have identified a series of seven structures belonging to angular furanocoumarin derivatives. The lattice energy of the identified structures has been calculated theoretically by PIXEL approach ${ }^{16}$. The theoretical calculation of the lattice energy of a crystal provides a better idea about the nature of crystal packing which corresponds to the experimentally calculated sublimation energy of the compound ${ }^{17}$. Molecular pairs were extracted from the crystal packing (after PIXEL calculation) and the nature and energy of the intermolecular interactions associated with the extracted molecular pairs was determined. A representative illustration of the coumarin moiety indicating the atomic numbering scheme used for the present work is shown in Figure 1. The chemical name, molecular code, chemical structure for each structure is presented in Table 1 and its precise crystallographic data in Table 2.<smiles>O=c1ccc2ccc3occc3c2o1</smiles>

Figure 1. Chemical structure of the furanocoumarin moiety and the numbering scheme used

Table 1. Chemical name, coding scheme and Chemical structure of the compounds

Chemical Name
Furo(2,3-h) coumarin ${ }^{18}$
(Angelicin)



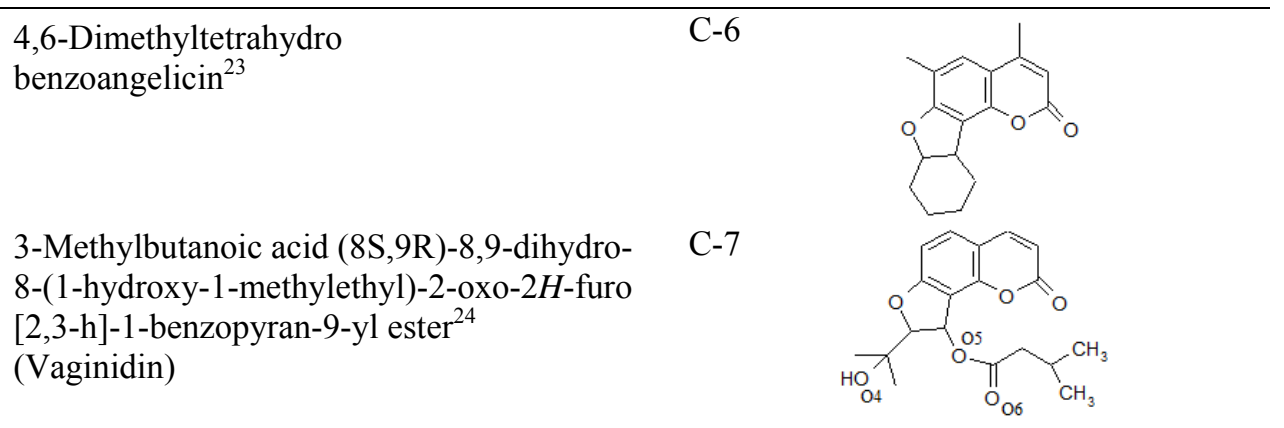

Table 2. Precise crystallographic data of angular furanocoumarin derivatives

\begin{tabular}{|c|c|c|c|c|c|c|c|}
\hline Data & $\mathrm{C}-1$ & C-2 & C-3 & C-4 & C-5 & C-6 & C-7 \\
\hline Formula & $\mathrm{C}_{11} \mathrm{H}_{6} \mathrm{O}_{3}$ & $\mathrm{C}_{14} \mathrm{H}_{12} \mathrm{O}_{3}$ & $\mathrm{C}_{13} \mathrm{H}_{10} \mathrm{O}_{5}$ & $\mathrm{C}_{18} \mathrm{H}_{16} \mathrm{O}_{5}$ & $\mathrm{C}_{17} \mathrm{H}_{10} \mathrm{O}_{3}$ & $\mathrm{C}_{17} \mathrm{H}_{16} \mathrm{O}_{3}$ & $\mathrm{C}_{19} \mathrm{H}_{22} \mathrm{O}_{6}$ \\
\hline $\begin{array}{l}\text { Mol. } \\
\text { Weight }\end{array}$ & 186.17 & 228.24 & 246.22 & 312.33 & 262.27 & 270.33 & 346.38 \\
\hline $\begin{array}{l}\text { Crystal } \\
\text { system }\end{array}$ & Orthorhombic & orthorhombic & Monoclinic & monoclinic & rhombohedral & tetragonal & orthorhombic \\
\hline $\begin{array}{l}\text { Space } \\
\text { group }\end{array}$ & $\mathrm{Pn}$ a $2_{1}$ & $\mathrm{P} 2_{1} 2_{1} 2_{1}$ & $\mathrm{P} 2{ }_{1} / \mathrm{n}$ & $\mathrm{P} 2{ }_{1} / \mathrm{n}$ & $\mathrm{R}-3$ & $\mathrm{P} 42 / \mathrm{n}$ & $\mathrm{P} 2_{1} 2_{1} 2_{1}$ \\
\hline $\mathrm{a}(\AA)$ & $11.527(9)$ & $5.210(1)$ & $90690(10)$ & $9.238(1)$ & $41.021(10)$ & $21.006(5)$ & $9.781(1)$ \\
\hline $\mathrm{b}(\AA)$ & $18.97(2)$ & $10.407(1)$ & $13.4984(5)$ & $10.959(1)$ & $41.021(10)$ & $21.006(5)$ & $17.328(6)$ \\
\hline $\mathrm{c}(\AA)$ & $3.781(5)$ & $20.478(2)$ & $20.9360(9)$ & $15.168(1)$ & $3.888(2)$ & $6.055(3)$ & $21.633(2)$ \\
\hline$\alpha\left({ }^{0}\right)$ & 90 & 90 & 90 & 90 & 90 & 90 & $90.0(10)$ \\
\hline$\beta\left(^{\circ}\right)$ & 90 & 90 & $91.733(2)$ & $90.64(1)$ & 90 & 90 & $90.0(10)$ \\
\hline$\gamma\left({ }^{0}\right)$ & 90 & 90 & 90 & 90 & 120 & 90 & $90.0(10)$ \\
\hline $\mathrm{R}$ & 0.057 & 0.037 & 0.0557 & 0.045 & 0.034 & 0.0662 & 0.078 \\
\hline
\end{tabular}

\section{Theoretical calculations}

The lattice energies of all the compounds have been calculated by PIXEL using the coulombLondon-Pauli (CLP) model of intermolecular coulombic, polarization, dispersion and repulsion energies ${ }^{16}$. For this purpose $\mathrm{H}$ atoms were moved to their neutron value. Two output files are generated after the end of the calculation. The first (.pri file) consists of the total lattice energies partitioned into their coulombic, polarization, dispersion and repulsion contributions (Table 3). The second (mlc file) consists of a molecule-molecule interaction energy along with the symmetry elements which relate to the molecules. The interaction energy of selected molecular pairs (from the .mlc file), extracted from the crystal packing along with the involved intermolecular interactions are listed in Table 4, with the total energies being partitioned into their coulombic, polarization, dispersion and repulsion contributions. The molecular pairs are arranged in decreasing order of their stabilizing energies. The geometrical restrictions placed on the intermolecular H-bonds present in the selected molecular pairs are the sum of the Vander Waals radii $+0.4 \AA$ and the directionality is greater than $110^{\circ}$.

\section{Results and Discussion}

\section{Furo(2,3-h) coumarin (C-1)}

All the molecular pairs (1-6) extracted from the crystal packing are shown in the Figure 2. The most stabilizing molecular pair shows the presence of $\mathrm{C}\left(\mathrm{sp}^{2}\right)-\mathrm{H} . . \mathrm{O}$ hydrogen bonding involving $\mathrm{H} 10$ with $\mathrm{O} 2$ and $\mathrm{H} 11$ with $\mathrm{O} 1$, having an interaction energy of $-6.21 \mathrm{kcal} \mathrm{mol}^{-1}$ (Figure 2, motif 1) with $45 \%$ contribution to stabilization from coulombic component. 

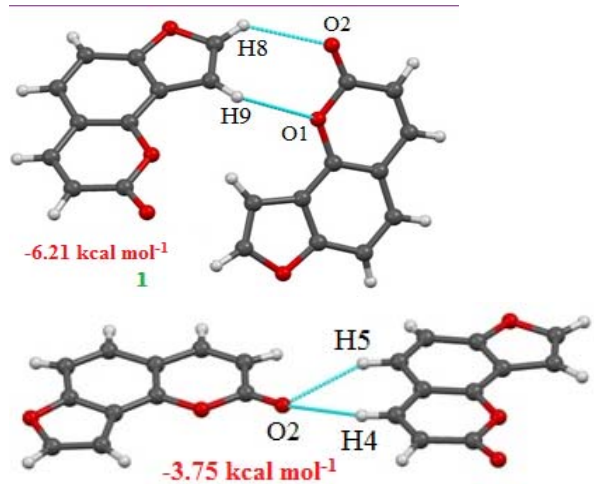

3

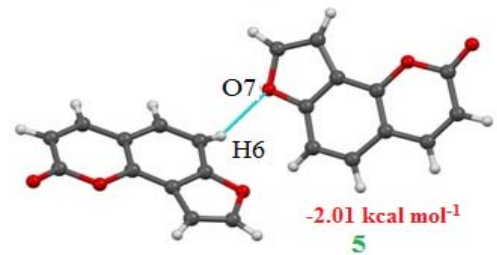

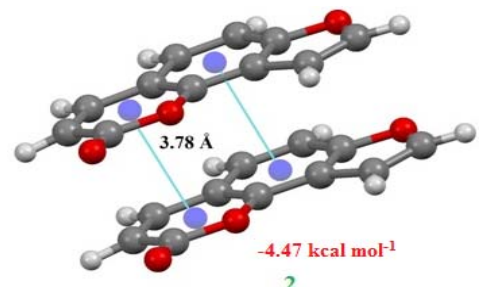
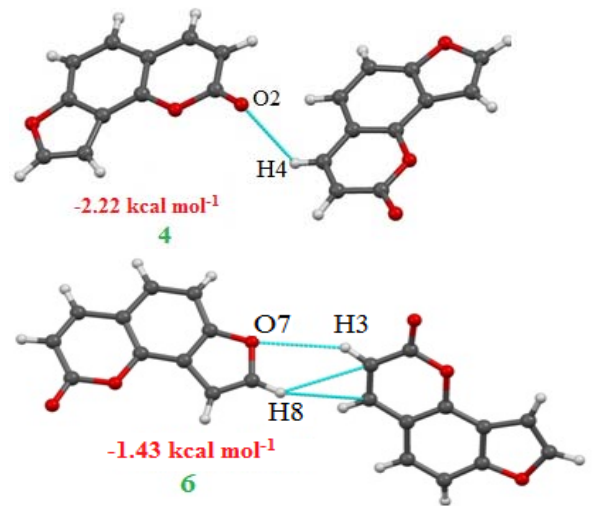

Figure 2. Molecular pairs (1-6) along with their interaction energies in C-1

Table 3. Lattice energy from CLP (in $\mathrm{kcal} \mathrm{mol}^{-1}$ )

\begin{tabular}{cccccc}
\hline Molecule & $\mathrm{E}_{\text {Cou }}$ & $\mathrm{E}_{\text {Pol }}$ & $\mathrm{E}_{\text {Disp }}$ & $\mathrm{E}_{\text {Rep }}$ & $\mathrm{E}_{\text {Tot }}$ \\
\hline C-1 & -8.60 & -3.11 & -24.66 & 15.46 & -20.93 \\
C-2 & -9.39 & -3.87 & -30.59 & 18.06 & -25.81 \\
C-3 & -11.12 & -4.30 & -30.21 & 18.59 & -27.10 \\
C-4 & -9.82 & -3.66 & -33.43 & 17.99 & -28.92 \\
C-5 & -8.77 & -4.01 & -34.5 & 19.41 & -27.91 \\
C-6 & -10.01 & -4.56 & -35.32 & 22.9 & -26.98 \\
C-7 & -14.98 & -6.02 & -34.29 & 22.37 & -32.91
\end{tabular}

Table 4. PIXEL interaction energies (I.E.) (kcal/mol) between molecular pairs related by a symmetry operation and the associated intermolecular interactions in the crystal

\begin{tabular}{|c|c|c|c|c|c|c|c|c|}
\hline Motif & $\begin{array}{c}\text { Centroid } \\
\text { Distance }(\AA)\end{array}$ & $\mathrm{E}_{\text {Coul }}$ & $\mathrm{E}_{\mathrm{Pol}}$ & $\mathrm{E}_{\text {Disp }}$ & $E_{\text {Rep }}$ & $\mathrm{E}_{\mathrm{Tot}}$ & Symmetry & $\begin{array}{l}\text { Important } \\
\text { interactions }\end{array}$ \\
\hline \multicolumn{9}{|c|}{ C-1 } \\
\hline 1 & 6.296 & -4.11 & -1.34 & -3.73 & 2.96 & -6.21 & $1-x, 1-y,-1 / 2+z$ & $\begin{array}{l}\mathrm{C} 10-\mathrm{H} 10 \ldots \mathrm{O} 2 \\
\mathrm{C} 11-\mathrm{H} 11 \ldots \mathrm{O} 1\end{array}$ \\
\hline 2 & 3.781 & -0.35 & -0.86 & -10.15 & 6.9 & -4.47 & $\mathrm{x}, \mathrm{y},-1+\mathrm{z}$ & $\pi \ldots \pi$ \\
\hline 3 & 8.945 & -2.77 & -0.98 & -2.08 & 2.05 & -3.75 & $\begin{array}{l}-1 / 2+x, 1 / 2-y,- \\
1+z\end{array}$ & $\begin{array}{l}\mathrm{C} 3-\mathrm{H} 3 \ldots \mathrm{O} 2 \\
\mathrm{C} 4-\mathrm{H} 4 \ldots \mathrm{O} 2\end{array}$ \\
\hline 4 & 8.107 & -0.55 & -0.59 & -2.34 & 1.26 & -2.22 & $1 / 2+x, 1 / 2-y, z$ & $\mathrm{C} 3-\mathrm{H} 3 \ldots \mathrm{O} 2$ \\
\hline 5 & 8.063 & -0.50 & -0.28 & -2.36 & 1.15 & -2.01 & $-\mathrm{x}, 1-\mathrm{y},-1 / 2+\mathrm{z}$ & C5-H5_..O3 \\
\hline 6 & 9.734 & -0.16 & -0.24 & -1.69 & 0.67 & -1.43 & $\begin{array}{l}1 / 2-x, 1 / 2+y,- \\
1 / 2+z\end{array}$ & $\begin{array}{l}\mathrm{C} 2-\mathrm{H} 2 \ldots \mathrm{O} 3 \\
\mathrm{C} 10-\mathrm{H} 10 \ldots \pi(\mathrm{C} 3, \mathrm{C} 4)\end{array}$ \\
\hline \multicolumn{9}{|c|}{$\mathrm{C}-2$} \\
\hline 1 & 5.210 & -1.67 & -1.17 & -9.11 & 5.74 & -6.21 & $-1+x, y, z$ & $\begin{array}{l}\text { Molecular stacking, } \\
\text { C11-H11b...O3, } \\
\text { C14-14c...C13, } \\
\text { H11b H1n }\end{array}$ \\
\hline
\end{tabular}




\begin{tabular}{|c|c|c|c|c|c|c|c|c|}
\hline & 5.831 & -1.48 & -0.96 & -5.16 & 2.63 & -4.99 & $\begin{array}{l}-1 / 2+x, 1 / 2- \\
y, 2-z\end{array}$ & $\begin{array}{l}\text { C11-H11b...O1, } \\
\text { C11-H11b...O2 }\end{array}$ \\
\hline 3 & 7.380 & -0.88 & -0.52 & -4.58 & 2.53 & -3.46 & $\begin{array}{l}1-x,-1 / 2+y \\
3 / 2-z\end{array}$ & $\begin{array}{l}\mathrm{C} 2-\mathrm{H} 2 \ldots \pi \\
\mathrm{C} 3-\mathrm{H} 3 \ldots \pi \\
\mathrm{C} 14-\mathrm{H} 14 \mathrm{a} \ldots \pi\end{array}$ \\
\hline 4 & 8.148 & -1.60 & -0.91 & -4.49 & 3.58 & -3.39 & $-\mathrm{x},-1 / 2+\mathrm{y}, 3 / 2-\mathrm{z}$ & $\begin{array}{l}\mathrm{C} 4-\mathrm{H} 4 \ldots \mathrm{O} 3 \\
\mathrm{H} 5 \ldots \mathrm{H} 14 \mathrm{a}\end{array}$ \\
\hline 5 & 11.638 & -1.31 & -0.38 & -1.94 & 0.91 & -2.72 & $-1+x,-1+y, z$ & C13-H13a...O2 \\
\hline 6 & 9.396 & -1.67 & -0.55 & -1.09 & 1.24 & -2.08 & $3 / 2+x, 1 / 2-y, 2-z$ & C13- H13b...O2 \\
\hline 7 & 12.095 & -0.09 & -0.12 & -1.84 & 0.78 & -1.24 & $-1 / 2+x,-1 / 2-y, 2-z$ & C13-H13a...C13 \\
\hline 8 & 10.407 & -.09 & -.09 & -1.46 & 0.64 & -1.00 & $\mathrm{x},-1+\mathrm{y}, \mathrm{z}$ & $\begin{array}{l}\mathrm{C} 14-\mathrm{H} 14 \mathrm{~b} \ldots \pi(\mathrm{C} 2, \\
\mathrm{C} 3)\end{array}$ \\
\hline \multicolumn{9}{|c|}{ C-3 } \\
\hline 1 & 8.007 & -7.72 & -2.31 & -5.21 & 4.71 & $-\overline{10.56}$ & $1-x,-y, 1-z$ & $\begin{array}{l}\mathrm{C} 10-\mathrm{H} 10 \ldots \mathrm{O} 2, \\
\mathrm{C} 11-\mathrm{H} 11 \ldots \mathrm{O} 1, \\
\mathrm{C} 11-\mathrm{H} 11 \ldots \mathrm{O} 2\end{array}$ \\
\hline 2 & 3.907 & -1.86 & -1.84 & -14.31 & 9.75 & -8.27 & $1+\mathrm{x}, \mathrm{y}, \mathrm{z}$ & $\begin{array}{l}\pi \ldots \pi, \\
\mathrm{C} 12-\mathrm{H} 12 \mathrm{c} \ldots \mathrm{O} 4 \\
\mathrm{C} 13-\mathrm{H} 13 \mathrm{c} \ldots \mathrm{O} 5\end{array}$ \\
\hline 3 & 8.023 & -2.10 & -0.74 & -4.09 & 2.17 & -4.71 & $-x, 1-y, 1-z$ & $\begin{array}{l}\mathrm{C} 2-\mathrm{H} 2 \ldots \mathrm{O} 4 \\
\mathrm{H} 3 \ldots \mathrm{H} 3\end{array}$ \\
\hline 4 & 8.422 & -1.15 & -0.62 & -4.56 & 2.34 & -3.99 & $1-x, 1-y, 1-z$ & $\begin{array}{l}\mathrm{C} 3-\mathrm{H} 3 \ldots \pi(\mathrm{C} 2, \mathrm{C} 3) \\
\mathrm{H} 2 \ldots \mathrm{H} 3\end{array}$ \\
\hline 5 & 10.594 & -1.86 & -0.50 & -1.67 & 1.6 & -2.44 & $\begin{array}{l}1 / 2+x, 1 / 2- \\
y, 1 / 2+z\end{array}$ & $\begin{array}{l}\text { C13-H13c...O2, } \\
\text { C13-H13a...O2 }\end{array}$ \\
\hline 6 & 11.844 & -1.46 & -0.38 & -0.74 & 0.67 & -1.91 & $\begin{array}{l}-3 / 2+x, 1 / 2- \\
y,-1 / 2+z\end{array}$ & C13-H13a...O2 \\
\hline 7 & 9.905 & -0.0 & -0.52 & -2.56 & 1.60 & -1.48 & $\begin{array}{l}-1 / 2-x,- \\
1 / 2+y, 1 / 2-z\end{array}$ & $\begin{array}{l}\mathrm{C} 12-\mathrm{H} 12 \mathrm{a} \ldots . . \mathrm{C} 13, \\
\mathrm{C} 10-\mathrm{H} 10 \ldots \mathrm{C} 13, \\
\mathrm{H} . . . \mathrm{H}\end{array}$ \\
\hline \multicolumn{9}{|c|}{ C-4 } \\
\hline 1 & 5.314 & -5.81 & -2.41 & -15.65 & $\begin{array}{l}11.6 \\
4\end{array}$ & $-\overline{12.24}$ & $1-x, 1-y, 1-z$ & $\begin{array}{l}\text { Molecular stacking, } \\
\text { C11-H11...O5, } \\
\text { C13-H13b...O1 }\end{array}$ \\
\hline 2 & 5.666 & -2.41 & -1.19 & -10.39 & 4.73 & -9.27 & $-x, 1-y, 1-z$ & $\begin{array}{l}\text { Molecular stacking, } \\
\text { C14-H14b...O2, } \\
\text { C15-H15...O2 }\end{array}$ \\
\hline 3 & 9.140 & -1.74 & -0.66 & -5.31 & 2.32 & -5.37 & $1-x, 2-y, 1-z$ & C16-H16a...O5 \\
\hline 4 & 9.274 & -0.57 & -0.74 & -5.54 & 3.13 & -3.70 & $\begin{array}{l}-1 / 2+x, 3 / 2- \\
y, 1 / 2+z\end{array}$ & $\begin{array}{l}\text { C18-H18b...O3, } \\
\text { Н...H }\end{array}$ \\
\hline 5 & 9.349 & -0.67 & -0.50 & -5.02 & 2.77 & -3.44 & $-\mathrm{x}, 2-\mathrm{y}, 1-\mathrm{z}$ & 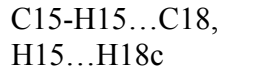 \\
\hline 6 & 10.959 & -1.41 & -0.55 & -2.25 & 1.19 & -3.01 & $\mathrm{x}, 1+\mathrm{y}, \mathrm{z}$ & $\begin{array}{l}\mathrm{C} 18-\mathrm{H} 18 \mathrm{a} \ldots \mathrm{OO} 2, \\
\mathrm{C} 11-\mathrm{H} 11 \ldots \mathrm{C} 16\end{array}$ \\
\hline 7 & 12.280 & -2.15 & -0.48 & -0.98 & 0.86 & -2.78 & $\begin{array}{l}-1 / 2+x, 1 / 2- \\
y, 1 / 2+z\end{array}$ & C10-H10_..O2 \\
\hline 8 & 9.176 & -0.28 & -0.19 & -3.68 & 1.86 & -2.29 & $\begin{array}{l}1 / 2-x, 1 / 2+ \\
y, 1 / 2-z\end{array}$ & $\begin{array}{l}\mathrm{C} 14-\mathrm{H} 14 \mathrm{a} \ldots \pi \\
\mathrm{C} 14-\mathrm{H} 14 \mathrm{~b} \ldots \pi\end{array}$ \\
\hline \multicolumn{9}{|c|}{ C-5 } \\
\hline 1 & 8.047 & -6.95 & -2.13 & -5.40 & 4.80 & -9.68 & $\begin{array}{l}2 / 3-x, 1 / 3-y,- \\
2 / 3-z\end{array}$ & $\begin{array}{l}\mathrm{C} 10-\mathrm{H} 10 \ldots \mathrm{O} 2 \\
\mathrm{C} 11-\mathrm{H} 11 \ldots \mathrm{O} 1\end{array}$ \\
\hline 2 & 3.888 & -0.55 & -1.22 & -15.11 & 9.15 & -7.74 & $\mathrm{x}, \mathrm{y},-1+\mathrm{z}$ & $\pi \ldots \pi$ \\
\hline
\end{tabular}




\begin{tabular}{|c|c|c|c|c|c|c|c|c|}
\hline 3 & 8.243 & -1.12 & -0.52 & -3.99 & 1.43 & -4.21 & $\begin{array}{l}2 / 3-\mathrm{x}, 1 / 3- \\
\mathrm{y}, 1 / 3-\mathrm{z}\end{array}$ & 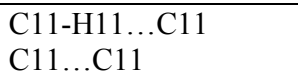 \\
\hline 4 & 10.217 & -1.69 & -0.64 & -2.94 & 1.88 & -3.39 & $\begin{array}{l}1 / 3+y, 2 / 3- \\
x+y,-4 / 3-z\end{array}$ & $\begin{array}{l}\mathrm{C} 14-\mathrm{H} 14 \ldots \mathrm{O} 2 \\
\mathrm{C} 15-\mathrm{H} 15 \ldots \mathrm{C} 16\end{array}$ \\
\hline 5 & 9.129 & -0.74 & -0.59 & -4.08 & 2.15 & -3.27 & $\begin{array}{l}2 / 3-x+y, 1 / 3- \\
x,-2 / 3+z\end{array}$ & $\begin{array}{l}\mathrm{C} 3-\mathrm{H} 3 \ldots \mathrm{O} 3, \\
\mathrm{C} 13-\mathrm{H} 13 \ldots \mathrm{O} 3, \\
\mathrm{C} 5-\mathrm{H} 5 \ldots \mathrm{C} 4, \\
\mathrm{H} 10 \ldots \mathrm{H} 13\end{array}$ \\
\hline 6 & 8.848 & -0.45 & -0.43 & -3.20 & 1.65 & -2.44 & $\begin{array}{l}1 / 3-y,-1 / 3+x- \\
y,-1 / 3+z\end{array}$ & $\begin{array}{l}\mathrm{C} 4-\mathrm{H} 4 \ldots \mathrm{O} 3, \\
\mathrm{C} 4-\mathrm{H} 4 \ldots \pi(\mathrm{C} 5, \mathrm{C} 6)\end{array}$ \\
\hline 7 & 9.350 & -0.52 & -0.31 & -2.79 & 1.43 & -2.19 & $\begin{array}{l}1 / 3+y, 2 / 3- \\
x+y,-1 / 3-z\end{array}$ & $\begin{array}{l}\text { C17-H17 ... (C14, } \\
\text { C15) } \\
\text { H15...H16 }\end{array}$ \\
\hline \multicolumn{9}{|c|}{ C-6 } \\
\hline 1 & 6.055 & -2.29 & -1.65 & -12.21 & 8.72 & -7.41 & $\mathrm{x}, \mathrm{y},-1+\mathrm{z}$ & $\begin{array}{l}\mathrm{C} 12-\mathrm{H} 12 \mathrm{~b} \ldots \mathrm{O} 2, \mathrm{C} 15- \\
\mathrm{H} 15 \mathrm{a} . . \mathrm{O} 1, \\
\text { Stacking, H16a...H7c }\end{array}$ \\
\hline 2 & 6.818 & -2.25 & -1.26 & -8.03 & 4.68 & -6.88 & $1-x, 1-y,-z$ & $\begin{array}{l}\text { C16-H16b...O2, } \\
\text { stacking }\end{array}$ \\
\hline 3 & 10.954 & -3.99 & -1.88 & -3.56 & 3.89 & -5.54 & $1-x, 1-y,-1-z$ & $\begin{array}{l}\mathrm{C} 2-\mathrm{H} 2 \ldots \mathrm{O} 2, \\
\mathrm{C} 16-\mathrm{H} 16 \mathrm{c} . . \mathrm{O} 2, \\
\mathrm{H} 2 \ldots \mathrm{H} 2\end{array}$ \\
\hline 4 & 8.155 & -2.13 & -1.07 & -7.19 & 5.42 & -4.95 & $\begin{array}{l}1 / 2+y, 1-x,- \\
1 / 2+z\end{array}$ & $\begin{array}{l}\mathrm{C} 4-\mathrm{H} 4 \ldots \mathrm{O} 3, \\
\mathrm{C} 16-\mathrm{H} 16 \mathrm{~b} \ldots \pi(\mathrm{C} 10, \\
\mathrm{C} 15)\end{array}$ \\
\hline 5 & 8.565 & -1.88 & -0.72 & -2.34 & 1.43 & -3.51 & $y, 1 / 2-x, 1 / 2-z$ & $\mathrm{C} 15-\mathrm{H} 15 \mathrm{~b} \ldots \mathrm{O} 2$ \\
\hline 6 & 10.481 & -0.45 & -0.31 & -3.11 & 1.93 & -1.93 & $1 / 2-y, x, 3 / 2-z$ & $\mathrm{C} 14-\mathrm{H} 14 \mathrm{a} \ldots \pi(\mathrm{C} 13)$ \\
\hline \multicolumn{9}{|c|}{ C-7 } \\
\hline A...B & 5.137 & -4.56 & -2.17 & -14.01 & 8.96 & -11.81 & $\begin{array}{l}1-\mathrm{x}, 1 / 2+\mathrm{y} \\
3 / 2-\mathrm{z}\end{array}$ & $\begin{array}{c}\pi \ldots \pi, \\
\mathrm{C} 10-\mathrm{H} 10 \ldots \mathrm{O} 2{ }^{\prime} \\
\mathrm{C} 10^{\prime}-\mathrm{H} 10^{\prime} \ldots \mathrm{O} 2 \\
\mathrm{C} 11-\mathrm{H} 11 \ldots \mathrm{O} 6{ }^{\prime}\end{array}$ \\
\hline A...B & 9.560 & -10.66 & -4.18 & -5.68 & $\begin{array}{c}10.6 \\
8\end{array}$ & -9.85 & $\mathrm{x}, \mathrm{y}, \mathrm{z}$ & $\begin{array}{c}\mathrm{O} 4^{\prime}-\mathrm{H}_{40}{ }^{\prime} \ldots \mathrm{O} 4 \\
\mathrm{C} 14^{\prime} \ldots \mathrm{H} 14 \mathrm{a}^{\prime} \ldots \mathrm{O} 4 \\
\mathrm{C} 13^{\prime}-\mathrm{H} 13 \mathrm{~b}^{\prime} \ldots \mathrm{O} 4\end{array}$ \\
\hline A...B & 5.955 & -1.57 & -0.72 & -10.63 & 4.75 & -8.17 & $1 / 2+x, 3 / 2-y, 2-z$ & $\begin{array}{c}\text { C19'-H19b'... } \\
\text { C19...Cg1 }\end{array}$ \\
\hline $\begin{array}{l}4 \\
\text { A...B }\end{array}$ & 9.011 & -1.65 & -0.72 & -5.40 & 2.67 & -5.11 & $-1+x, y, z$ & $\begin{array}{c}\mathrm{C} 5-\mathrm{H} 5 \ldots \mathrm{O} 3^{\prime} \\
\mathrm{C} 14^{\prime}-\mathrm{H} 14 \mathrm{a}^{\prime} \ldots \pi \\
\left(\mathrm{C} 4^{\prime}, \mathrm{C} 5^{\prime}\right)\end{array}$ \\
\hline $\begin{array}{l}5 \\
\mathrm{~B} \ldots \mathrm{B}\end{array}$ & 9.781 & -1.98 & -0.74 & -3.61 & 1.36 & -4.97 & $-1+\mathrm{x}, \mathrm{y}, \mathrm{z}$ & $\begin{array}{l}\mathrm{C} 3^{\prime}-\mathrm{H} 3^{\prime} \ldots \mathrm{O}^{\prime} \\
\mathrm{C} 4^{\prime}-\mathrm{H} 4^{\prime} \ldots 5^{\prime}\end{array}$ \\
\hline $\begin{array}{l}6 \\
\text { A...A }\end{array}$ & 10.547 & -2.19 & -0.72 & -4.01 & 2.32 & -4.61 & $\begin{array}{c}2-\mathrm{x},-1 / 2+ \\
\mathrm{y}, 3 / 2-\mathrm{z}\end{array}$ & $\begin{array}{c}\mathrm{C} 5-\mathrm{H} 5 \ldots \mathrm{O} 2 \\
\mathrm{C} 2-\mathrm{H} 2 \ldots \mathrm{O} 3 \\
\mathrm{C} 14-\mathrm{H} 14 \mathrm{a} \ldots \pi(\mathrm{C} 2, \mathrm{C} 3)\end{array}$ \\
\hline $\begin{array}{l}7 \\
\text { A...A }\end{array}$ & 9.781 & -1.72 & -0.76 & -4.46 & 2.48 & -4.47 & $-1+\mathrm{x}, \mathrm{y}, \mathrm{z}$ & C3-H3 ...O6 \\
\hline $\begin{array}{l}8 \\
\text { A...B }\end{array}$ & 10.926 & -2.61 & -0.69 & -2.15 & 1.09 & -4.34 & $1+x, 1+y, z$ & $\begin{array}{c}\mathrm{C} 2-\mathrm{H} 2 \ldots \mathrm{O} 2^{\prime} \\
\mathrm{C} 2^{\prime}-\mathrm{H} 22^{\prime} \ldots \mathrm{O} 2\end{array}$ \\
\hline $\begin{array}{l}9 \\
\text { A...A } \\
\end{array}$ & 10.088 & -1.19 & -0.79 & -3.84 & 2.94 & -2.89 & $\begin{array}{c}1-\mathrm{x},- \\
1 / 2+\mathrm{y}, 3 / 2-\mathrm{z}\end{array}$ & $\begin{array}{l}\mathrm{C} 14-\mathrm{H} 14 \mathrm{c} . . . \mathrm{O} 6 \\
\mathrm{C} 13-\mathrm{H} 13 \mathrm{~b} \ldots \mathrm{O} 2\end{array}$ \\
\hline
\end{tabular}




\begin{tabular}{|c|c|c|c|c|c|c|c|c|}
\hline $\begin{array}{l}10 \\
\text { B...B }\end{array}$ & 10.212 & -0.38 & -0.33 & -3.54 & 1.43 & -2.82 & $\begin{array}{c}-1 / 2+\mathrm{x}, 3 / 2- \\
\mathrm{y}, 2-\mathrm{z}\end{array}$ & $\begin{array}{c}\mathrm{C} 14^{\prime}-\mathrm{H} 14 \mathrm{~b}^{\prime} \ldots \mathrm{C} 5^{\prime} \\
\mathrm{H} 13 \mathrm{c}^{\prime} \ldots \mathrm{H} 5^{\prime}\end{array}$ \\
\hline $\begin{array}{l}11 \\
\text { A...B }\end{array}$ & 10.11 & -0.43 & -0.28 & -2.36 & 0.97 & -2.10 & $-x, 1 / 2+y, 3 / 2-z$ & $\begin{array}{l}\mathrm{C} 2^{\prime}-\mathrm{H} 2^{\prime} \ldots \mathrm{C} 13 \\
\mathrm{C} 3^{\prime}-\mathrm{H} 3^{\prime} \ldots \mathrm{C} 13\end{array}$ \\
\hline $\begin{array}{l}12 \\
\text { B...B }\end{array}$ & 11.014 & -0.12 & -0.33 & -1.84 & 1.03 & -1.26 & $1 / 2+x, 1 / 2-y, 2-\bar{z}$ & $\mathrm{C} 19^{\prime}-\mathrm{H} 19 \mathrm{a}^{\prime} \ldots \mathrm{O} 2^{\prime}$ \\
\hline
\end{tabular}

$A$ and $B$ refers to two molecules in the asymmetric unit cell while 'indicate the second crystallographically independent molecule

The second most stabilized molecular pair in the crystal structure, formed via $\pi \ldots \pi$ molecular offsets $(\mathrm{Cg} 1$ and $\mathrm{Cg} 2$ with $\mathrm{Cg} 1$ and $\mathrm{Cg} 2$ of another molecule, where $\mathrm{Cg} 1$ is centroid of pyrone ring and $\mathrm{Cg} 2$ is centroid of benzene ring, centroid to centroid distance is $3.78 \AA$ ) along the crystallographic c axis, has a contribution of $-4.47 \mathrm{kcal} \mathrm{mol}^{-1}(90 \%$ contribution to stabilization from the dispersion energy) to the stabilization of the packing. These molecular stacks are interlinked via weak $\mathrm{C}\left(\mathrm{sp}^{2}\right)-\mathrm{H} \ldots \mathrm{O}$ intermolecular hydrogen bonds involving bifurcated acceptor atom $\mathrm{O} 2$ with $\mathrm{H} 3$ and $\mathrm{H} 4$ ( motif 3, $-3.75 \mathrm{kcal} \mathrm{mol}^{-1}$ ), $\mathrm{H} 3$ with $\mathrm{O} 2$ (motif 4, -2.22 $\mathrm{Kcal} \mathrm{mol}^{-1}$ ) and $\mathrm{H} 5$ with $\mathrm{O} 3$ (motif 5, -2.01 kcal mol ${ }^{-1}$ ) forming a layered arrangement down the ac plane as shown in Figure $3 \mathrm{a}$. However in the bc plane, the adjacent stacks are interconnected via motif $6\left(-1.43 \mathrm{Kcal} \mathrm{mol}^{-1}\right)$ showing the presence of $\mathrm{C}\left(\mathrm{sp}^{2}\right)-\mathrm{H} 2 \ldots \mathrm{O} 3$ and $\mathrm{C}\left(\mathrm{sp}^{2}\right)-\mathrm{H} 10 \ldots \pi$ intermolecular contacts (Figure $3 b$ ).

(a)
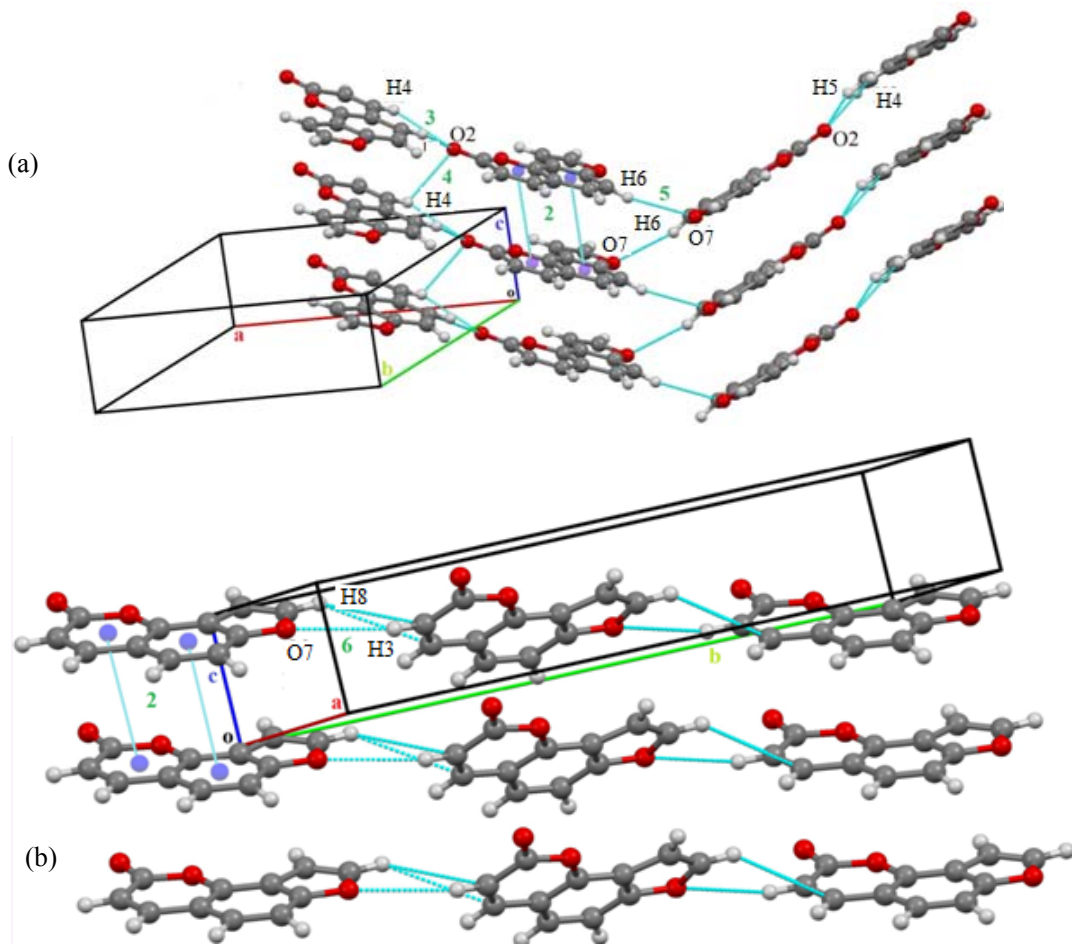

Figure 3. Packing view of the molecules depicting aromatic stacking interactions in C-1 down the (a) ac plan (b) bc plan

8,9-Dihydro-8-(1-methyl ethenyl)-2H-furo[2,3-h] -1- benzopyran-2-one (C-2)

All the molecular pairs (1-8) extracted after the PIXEL calculation are represented in Figure 4 along with their interaction energies. Molecules are stacked along the crystallographic a axis 
utilizing motif 1 . Motif 1 is the most stabilized pair with an interaction energy of $-6.21 \mathrm{kcal} \mathrm{mol}^{-1}$ in the crystal packing and consist of weak C-H...O (involving H11b with O3) and molecular stacking along with $\mathrm{C}-\mathrm{H} \ldots \mathrm{C}$ and $\mathrm{H}$...H interaction. The nature of the combined interaction is predominately dispersive in nature $(75 \%$ contribution to total stabilization comes from dispersion energy). Adjacent stacks are then interlinked via motif 5 and 8 (Figure 5). Motif 5 (I.E. $=-2.72 \mathrm{kcal} \mathrm{mol}^{-1}$ ) involves the presence of weak C-H...O (involving $\mathrm{H} 13 \mathrm{a}$ with $\mathrm{O} 2$ ) whereas the presence of $\mathrm{C}-\mathrm{H} . . . \pi$ links the molecules in motif 8 (I.E. $\left.=-1 \mathrm{kcal} \mathrm{mol}^{-1}\right)$. The second most stabilized pair involves the interaction of bifurcated donor $\mathrm{H} 11 \mathrm{~b}$ with acceptor atoms $\mathrm{O} 1$ and $\mathrm{O} 2$, contributing $-4.99 \mathrm{kcal} \mathrm{mol}^{-1}$ towards crystal stability. Another molecular pair shows the presence of $\mathrm{C}-\mathrm{H} . . . \pi$ hydrogen bond (motif 3 , Figure 4) having an interaction energy of $-3.46 \mathrm{kcal} \mathrm{mol}^{-1}$. Motif 4 (I.E. $=-3.39 \mathrm{kcal} \mathrm{mol}^{-1}$ ) involves the presence of $\mathrm{C}-\mathrm{H}$...O (involving $\mathrm{H} 4$ with $\mathrm{O} 3$ ) along with $\mathrm{H}$...H interaction whereas another $\mathrm{C}-\mathrm{H}$...O interaction links the molecules in motif $6\left(\mathrm{I} . \mathrm{E} .=-2.08 \mathrm{kcal} \mathrm{mol}^{-1}\right)$. Furthermore, additional stabilization of $-1.24 \mathrm{kcal} \mathrm{mol}^{-1}$ was also observed to be provided by motif 7 .

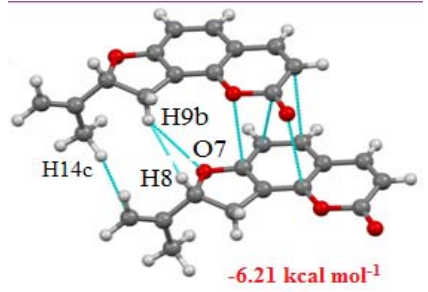

1
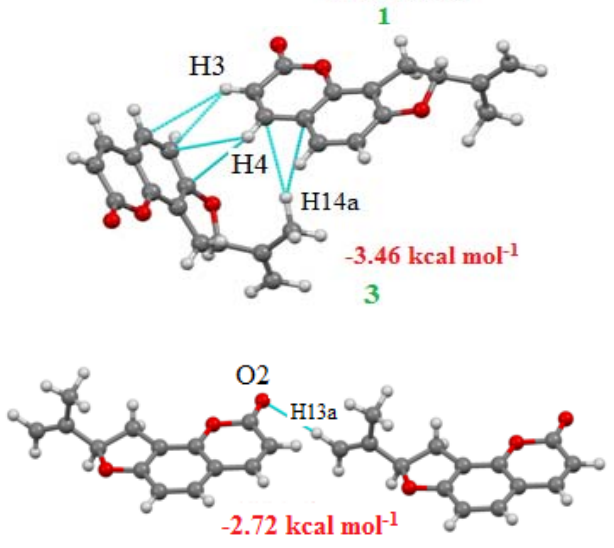

5
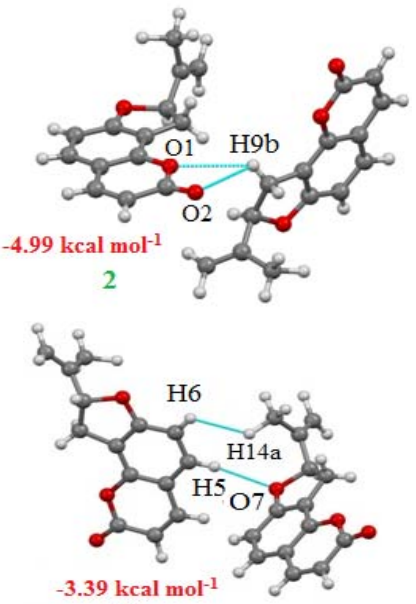

4

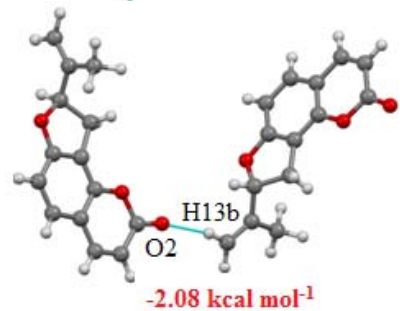

6
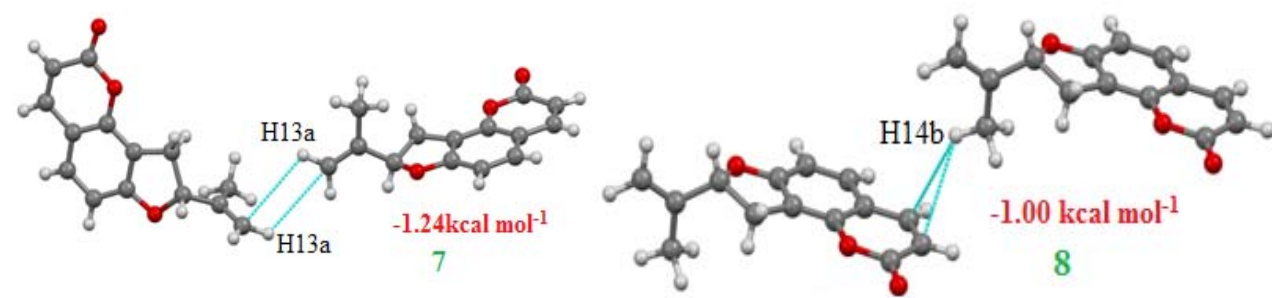

Figure 4. Molecular pairs (1-8) along with their interaction energies in C-2 


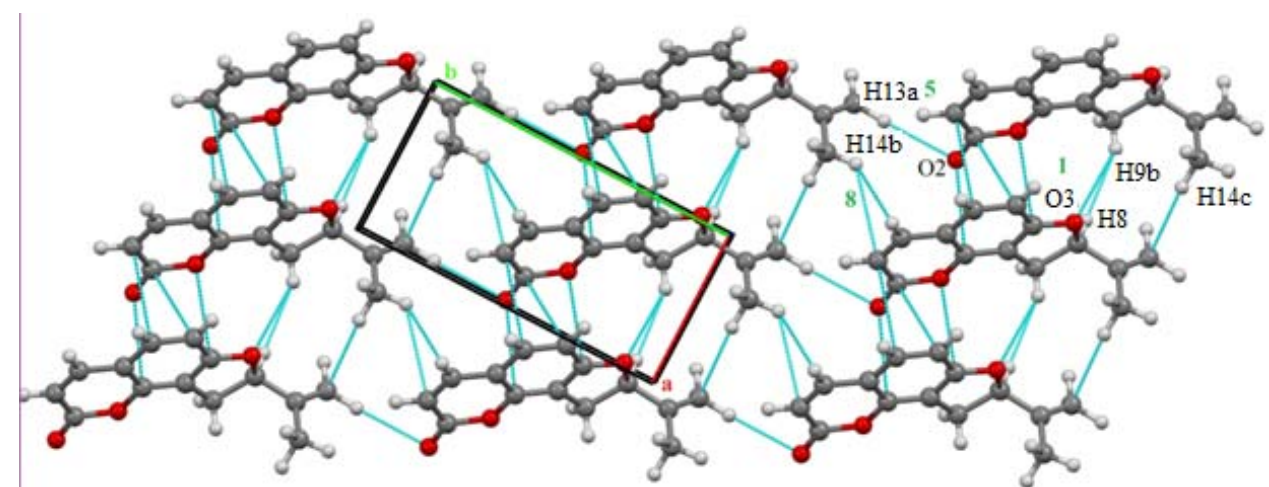

Figure 5. Packing of molecules in C-2 showing stacking of the molecules down the ab plane Syn-5,6-dimethoxy-2H-furo(2,3-h)-1-benzopyran-2-one (C-3)

The principal packing motifs (1-8) along with their respective interaction energies are shown in Figure 6. In this compound, the maximum stabilization comes from weak $\mathrm{C}\left(\mathrm{sp}^{2}\right)-\mathrm{H}$...O interactions, involving bifurcated donor $\mathrm{H} 11$ with $\mathrm{O} 1$ and $\mathrm{O} 2$ and $\mathrm{H} 10$ with bifurcated acceptor $\mathrm{O} 2$, generating dimers in the crystallographic bc plane acting across the centre of symmetry. The energy stabilization of this dimer is $-10.56 \mathrm{Kcal} \mathrm{mol}^{-1}$ as obtained from energy calculation performed using PIXEL. One striking feature of this dimer is that the share of coloumbic component in the total stabilization is $50 \%$. These dimers are then interconnected via motif $4\left(\mathrm{C}\left(\mathrm{sp}^{2}\right)-\mathrm{H} 3 \ldots \pi\right.$, I.E. $\left.=-3.99 \mathrm{kcal} \mathrm{mol}^{-1}\right)$ and motif 5 (weak C$\mathrm{H}$... O hydrogen bonds involving $\mathrm{H} 13 \mathrm{a}$ and $\mathrm{H} 13 \mathrm{c}$ with $\mathrm{O} 2$ being the acceptor atom, I.E. = $2.44 \mathrm{kcal} \mathrm{mol}^{-1}$ ) forming sheets in the bc plane (Figure 7a). The second most stabilized molecular pair in the crystal formed via $\pi \ldots \pi$ stacking interaction along with weak C-H...O interaction (involving H12c with O4 and H13c with O5) has a contribution of $-8.27 \mathrm{kcal} \mathrm{mol}^{-1}$ (with major dispersion contribution) to the stabilization of the crystal packing. Adjacent stacks are connected via weak $\mathrm{C}-\mathrm{H}$...O hydrogen bonds (motif 5 and motif 6 with stabilization energies being -2.44 and $-1.91 \mathrm{Kcal} \mathrm{mol}^{-1}$ ) in the ac plane (Figure $7 \mathrm{~b}$ ). However in the ab plane, molecular stacks formed by motif 2 are connected via motif 3 (dimeric $\mathrm{C}\left(\mathrm{sp}^{2}\right)-\mathrm{H} 2 \ldots \mathrm{O} 4$ along with $\mathrm{H} 3 \ldots \mathrm{H} 3$ interaction, I.E. $\left.=-4.71 \mathrm{kcal} \mathrm{mol}^{-1}\right)$ motif $4(-3.99 \mathrm{kcal}$ $\left.\mathrm{mol}^{-1}\right)$ and motif $7\left(-1.48 \mathrm{kcal} \mathrm{mol}^{-1}\right)$ forming layers (Figure $\left.7 \mathrm{c}\right)$.
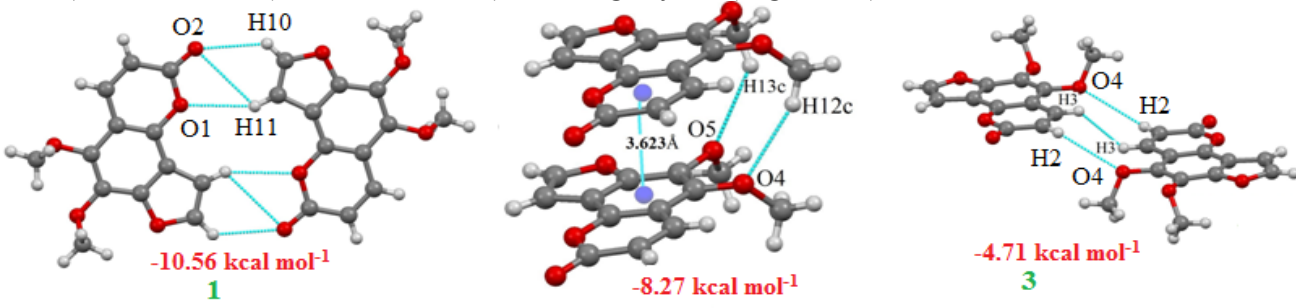

2
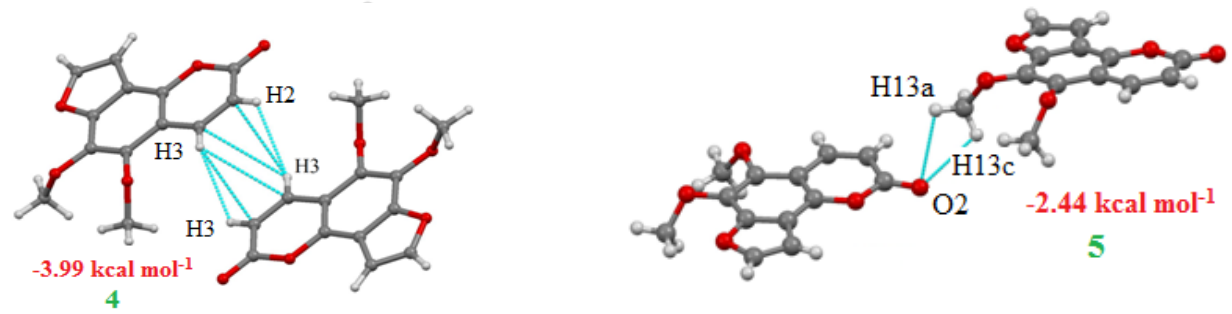

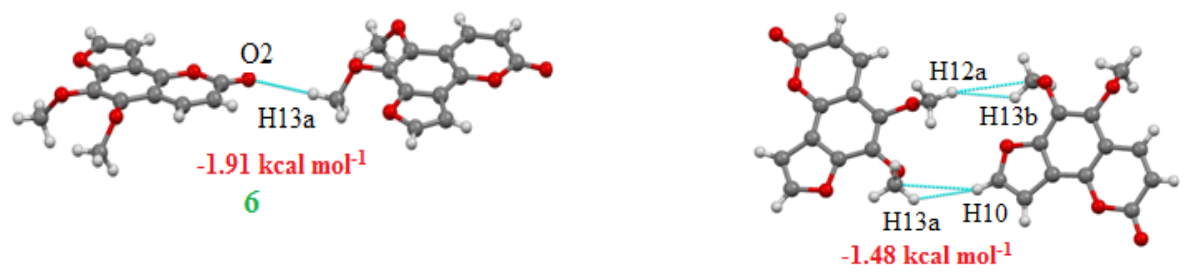

7

Figure 6. Molecular pairs (1-7) along with their interaction energies in C-3

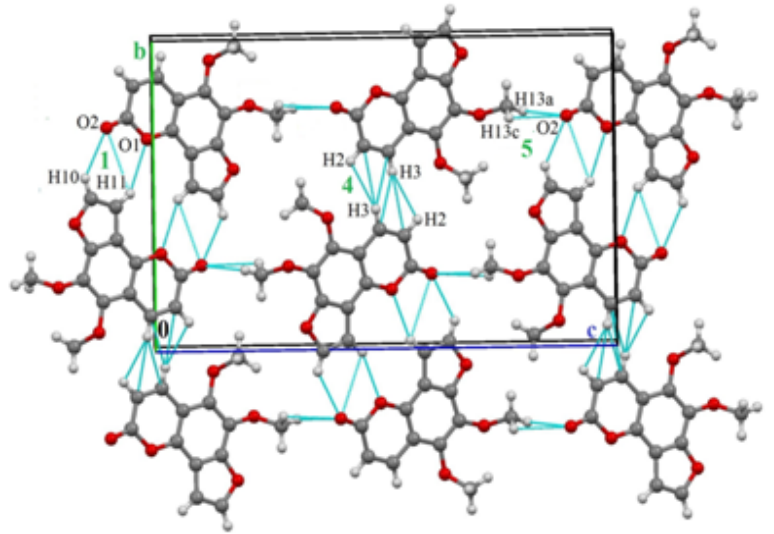

(a)

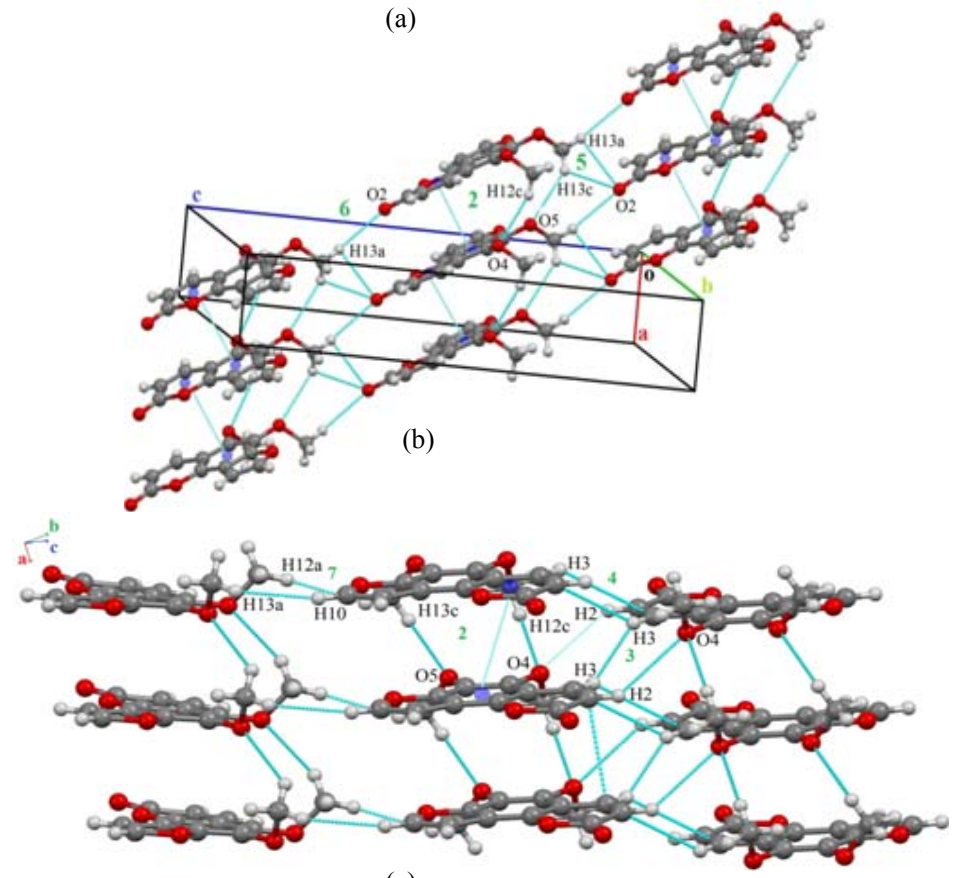

Figure 7. Packing of the molecules in C-3 viewed down (a) bc plane (b) ac plane and (c) ab plane 5-Acetoxy-6-(1,1-dimethyl-2- propenyl)-2H-furo(2,3-h)(1) benzopyran-2-one (C-4) The molecular pairs (1-8) extracted from the crystal packing which provide significant contribution towards the stabilization of the crystal are shown in Figure 8. The characteristic 
packing feature in this molecule consist of molecular stacking along with weak C-H...O hydrogen bonds. The key packing motifs $(1,2)$ in this structure shows the presence of molecular stacking along with weak C-H...O hydrogen bonds involving H11, H13b with O5 and $\mathrm{O} 1$ (motif 1, interaction energy(I.E) being $-12.24 \mathrm{kcal} \mathrm{mol}^{-1}$ ) and bifurcated acceptor atom $\mathrm{O} 2$ with $\mathrm{H} 14 \mathrm{~b}$ and $\mathrm{H} 15$ (motif 2, I.E $=-9.27 \mathrm{kcal} \mathrm{mol}^{-1}$ ). The combined nature of the interaction in these two pairs is dispersive in nature with $65 \%$ (motif 1 ) and $75 \%$ (motif 2 ) of contribution from dispersion component. These two structural motifs $(1,2)$ are stacked alternatively along the crystallographic a axis. Adjacent stacks are then interconnected via motif 3(dimeric C-H...O), 5 and 6 having energies $-5.37,-3.44$ and $-3.01 \mathrm{kcal} \mathrm{mol}^{-1}$ respectively (Figure. $9 \mathrm{~b}$ ). The packing in the crystal also displays the formation of molecular chain via $\mathrm{C}\left(\mathrm{sp}^{3}\right)-\mathrm{H} 18 \mathrm{a} \ldots \mathrm{O} 2$ along with $\mathrm{C}\left(\mathrm{sp}^{2}\right)-\mathrm{H} 11-\mathrm{C} 16$ (motif $6, \mathrm{I} . \mathrm{E}=-3.01 \mathrm{Kcal} \mathrm{mol}^{-1}$ ) along $\mathrm{b}$ - axis. The molecular chains are then interlinked by more stabilized motif $4\left(\mathrm{C}\left(\mathrm{sp}^{3}\right)\right.$ $\mathrm{H} 18 \mathrm{~b} . . \mathrm{OO} 3$ along with $\mathrm{H} . . \mathrm{H}$ interaction, I.E. $\left.=-3.7 \mathrm{Kcal} \mathrm{mol}^{-1}\right)$ and motif $7\left(\mathrm{C}\left(\mathrm{sp}^{2}\right)-\right.$ $\mathrm{H} 10 \ldots \mathrm{O} 2$, I.E. $=-2.78 \mathrm{Kcal} \mathrm{mol}^{-1}, 60 \%$ contribution from coulombic component) forming sheets in the bc plane (Figure. 9a). However, it is observed that motif 8 showing C-H... $\pi$ also provides additional stabilization of $-2.29 \mathrm{kcalmol}^{-1}$.
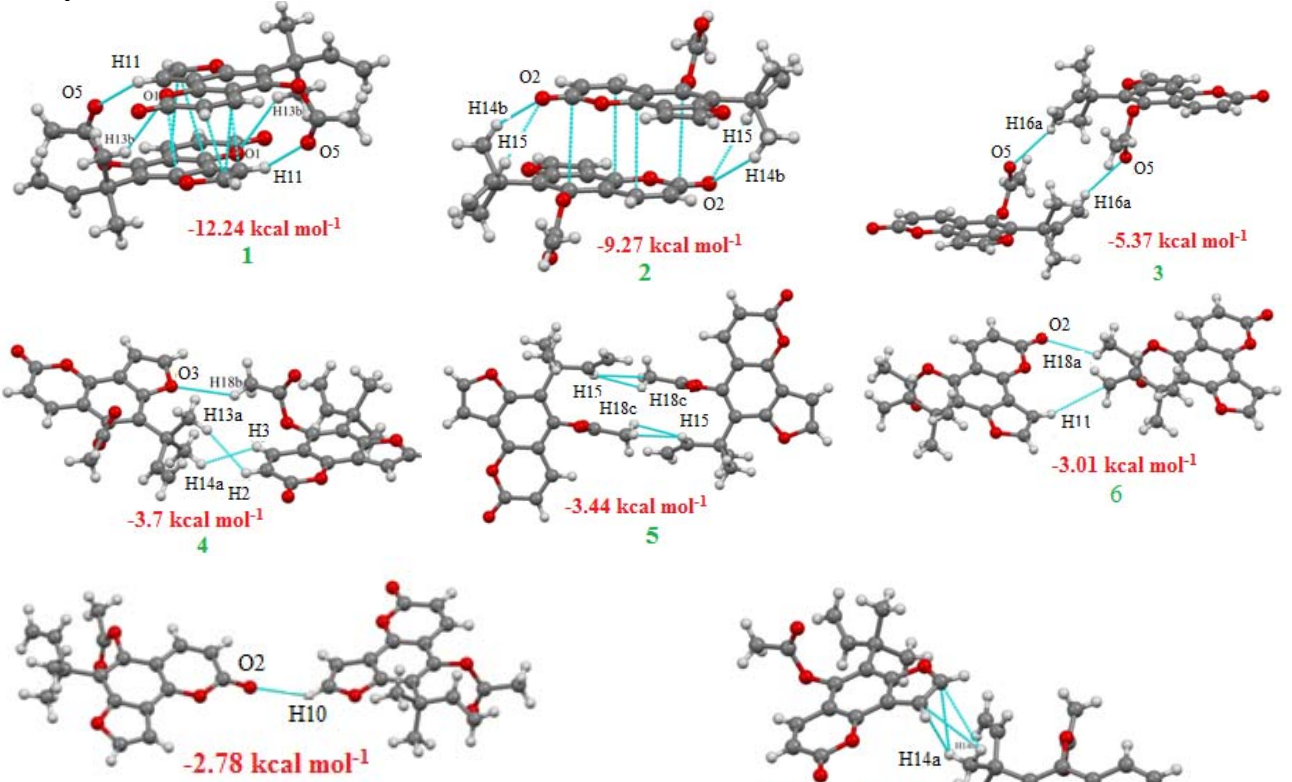

7

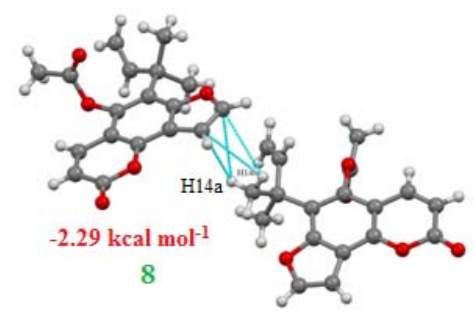

Figure 8. Molecular pairs (1-8) along with their interaction energies in C-4

\section{3-Phenyl-2H-furo(2,3-h)-1- benzopyran-2-one (C-5)}

The important packing motifs (1-7) extracted from crystal packing along with their stabilization energies are shown in Figure 10. The molecules are packed with the involvement of weak dimeric $\mathrm{C}\left(\mathrm{sp}^{2}\right)-\mathrm{H}$... O interaction involving $\mathrm{H} 11, \mathrm{H} 10$ with $\mathrm{O} 1$ and $\mathrm{O} 2$ (motif 1, I.E. $=-9.68 \mathrm{Kcal} \mathrm{mol}^{-1}$, about $50 \%$ contribution from coulombic component) and $\mathrm{H} 3, \mathrm{H} 13$ with bifurcated acceptor atom $\mathrm{O} 3$ along with $\mathrm{C}-\mathrm{H} . . . \pi$ interaction (motif 5, I.E. $=-3.27 \mathrm{Kcal} \mathrm{mol}^{-1}$ ), forming molecular zig-zag chains along the crystallographic $\mathrm{b}$ axis. The zig-zag chains thus formed are interconnected via motif 2, 3 and 6 (Figure 11). 
Molecular pair 2 involves $\pi \ldots \pi$ stacking, contributing $-7.74 \mathrm{Kcal} \mathrm{mol}^{-1}$ towards stabilization. One striking feature of consideration is that out of the total stabilization of $-16.88 \mathrm{kcal} \mathrm{mol}^{-1}$, $90 \%$ of stabilization is imparted by dispersion component. Molecular pair 3 shows C-H...C interaction $\left(-4.21 \mathrm{kcal} \mathrm{mol}^{-1}\right)$ whilst the presence of $\mathrm{C}-\mathrm{H} . . \mathrm{O}$ and $\mathrm{C}-\mathrm{H} \ldots \pi$ hydrogen bond links the molecule in motif $6\left(-2.44 \mathrm{Kcal} \mathrm{mol}^{-1}\right)$. Moreover, the presence of motif 4 (I.E $=$ $-3.39 \mathrm{kcal} / \mathrm{mol}$ ) which involves $\mathrm{C}-\mathrm{H} . . . \mathrm{O}($ involving $\mathrm{H} 14$ with $\mathrm{O} 2)$ and $\mathrm{CH} \cdots \pi$ interactions and motif $7(\mathrm{I} . \mathrm{E}=-2.84 \mathrm{kcal} / \mathrm{mol})$ showing weak $\mathrm{C}-\mathrm{H} \cdots \pi$ hydrogen bond along with $\mathrm{H} \ldots \mathrm{H}$ interaction were also observed to stabilize the crystal packing.

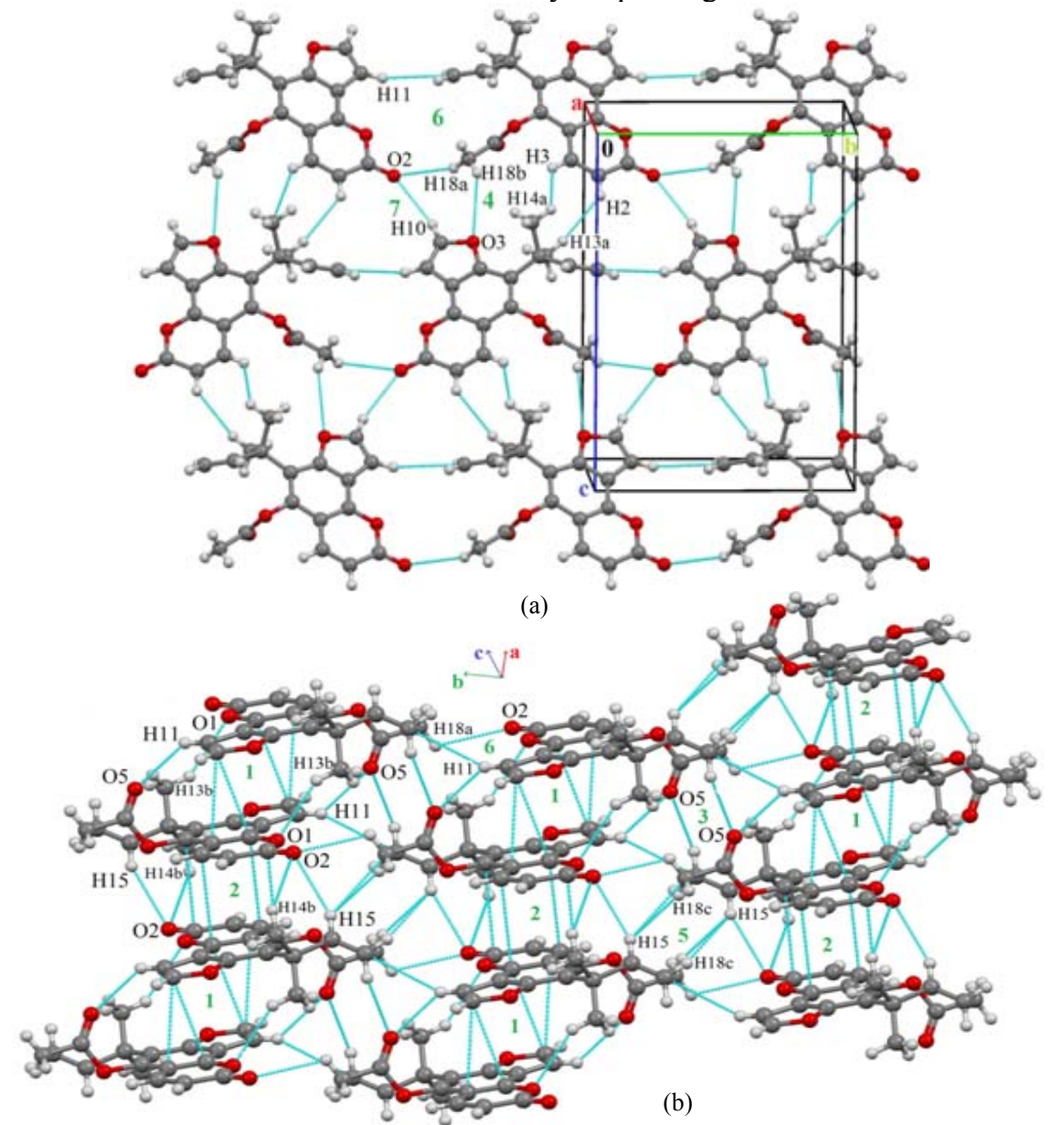

Figure 9. Packing of the molecules in C-4 viewed down (a) bc plane (b) ab plane
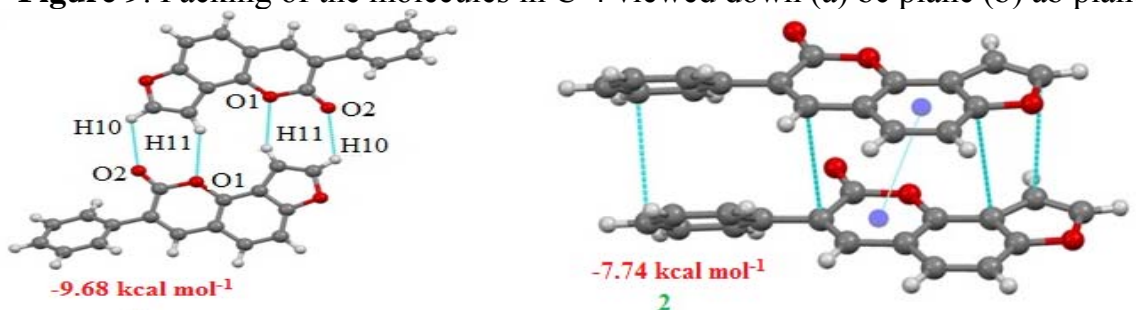

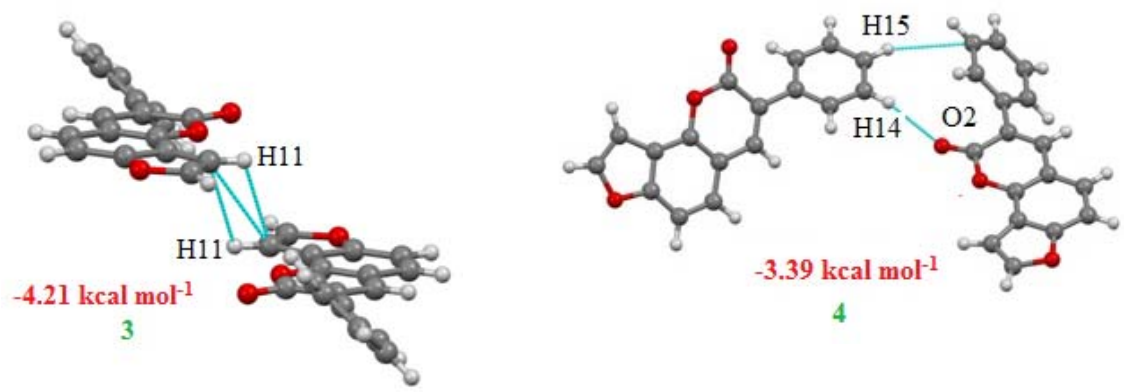

4
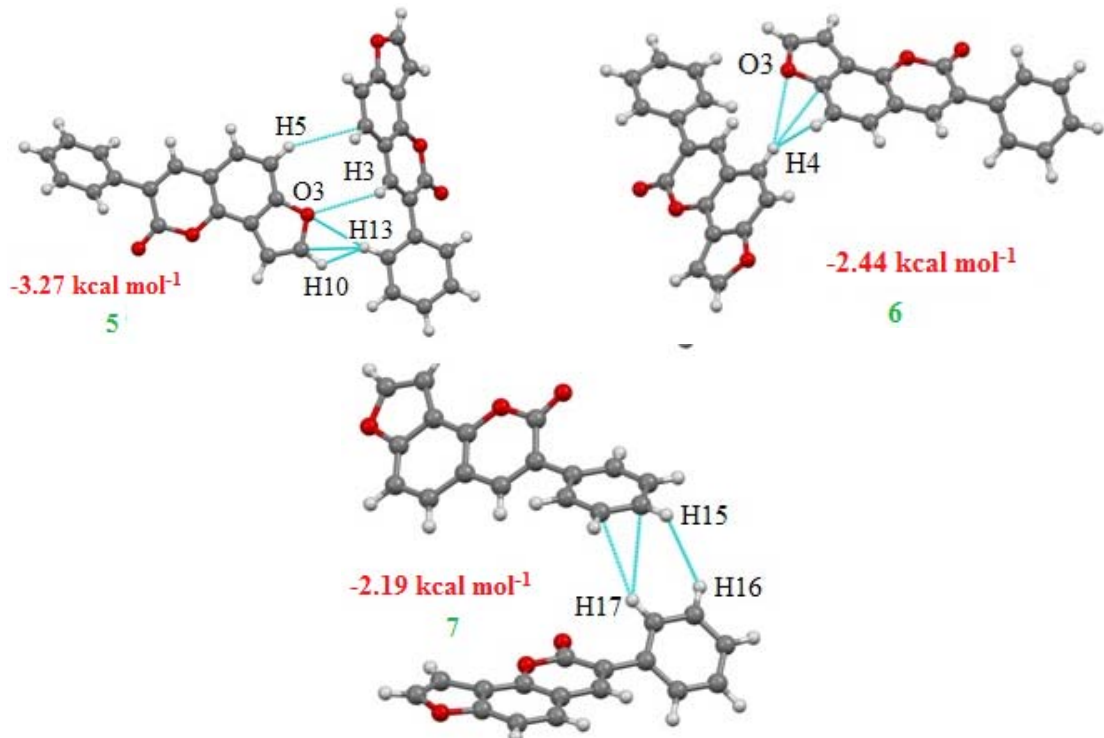

Figure 10. Molecular pairs (1-8) along with their interaction energies in C-5

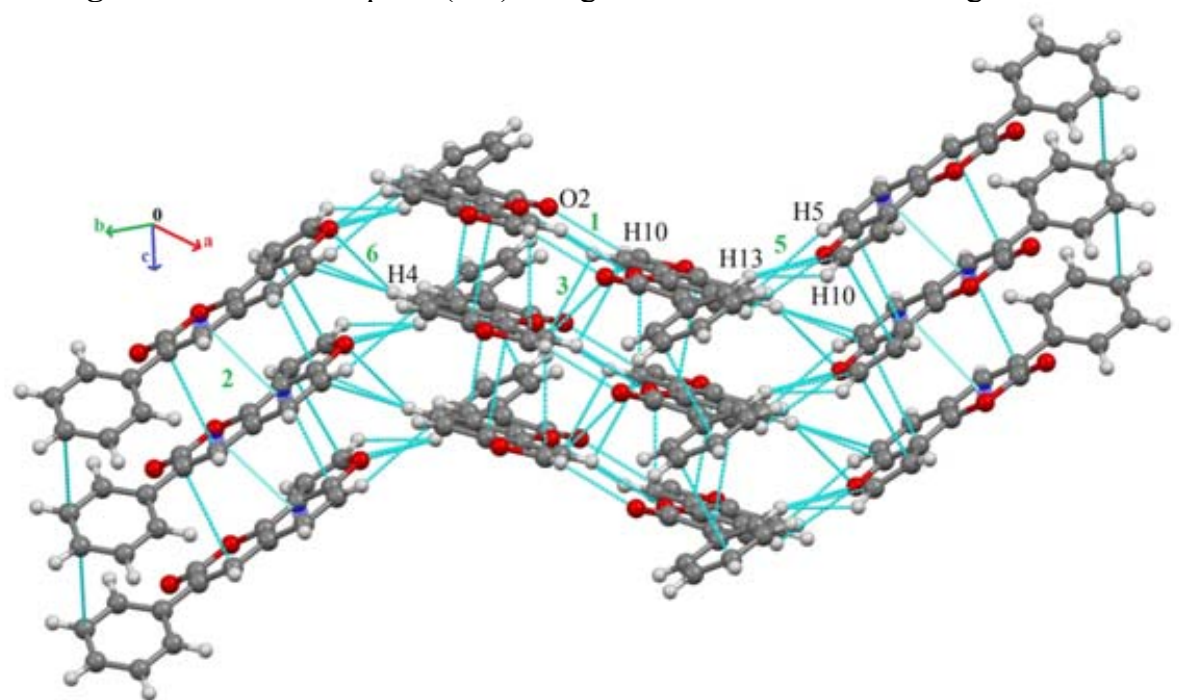

Figure 11. Crystal packing depicting zig-zag arrangement of molecules in C-5 


\section{Dimethyltetrahydro benzoangelicin (C-6)}

The important molecular pairs (1-6) extracted from the molecular packing are shown in Figure 12. The molecules in this structure are stacked along the crystallographic $b$ axis utilizing weak $\mathrm{C}-\mathrm{H} . . . \mathrm{O}$ interaction involving $\mathrm{H} 15 \mathrm{a}$ with $\mathrm{O} 1$ and $\mathrm{H} 12 \mathrm{~b}$ with $\mathrm{O} 2$ along with stacking interaction $(\mathrm{C} 10-\mathrm{C} 1$ with $\mathrm{C} . . \mathrm{C}$ distance being $3.42 \AA)$ and $\mathrm{C}-\mathrm{H} . . \mathrm{H}-\mathrm{C}$ interaction (motif 1). The stabilization energy of this pair is $-7.41 \mathrm{kcal} \mathrm{mol}^{-1}$ as obtained from energy calculation performed using PIXEL. These molecular stacks are then linked via motif 2 (involving dimeric $\mathrm{C}\left(\mathrm{sp}^{3}\right)-\mathrm{H} 16 \mathrm{~b} \ldots \mathrm{O} 2$ along with stacking interaction $\mathrm{C} 3 \ldots \mathrm{C} 2$ with $\mathrm{C} \ldots \mathrm{C}$ distance being $3.385 \AA$ ) and motif 3 (dimeric $\mathrm{C}\left(\mathrm{sp}^{3}\right)-\mathrm{H} \ldots \mathrm{O}$ hydrogen bonds involving $\mathrm{H} 16 \mathrm{c}$ and $\mathrm{H} 2$ with bifurcated acceptor atom $\mathrm{O} 2$ along with $\mathrm{C}\left(\mathrm{sp}^{2}\right)-\mathrm{H} \ldots \mathrm{H}-\mathrm{C}\left(\mathrm{sp}^{2}\right)$ with $\mathrm{H} . . . \mathrm{H}$ distance being $2.194 \AA$ which is less than the sum of their vander waal radii indicating the interaction between the two atoms) having energies -6.88 and $-5.54 \mathrm{Kcal} \mathrm{mol}^{-1}$ respectively, forming a layered arrangement in the bc plane (Figure 13). Motif 4 involves the presence of weak $\mathrm{C}\left(\mathrm{sp}^{2}\right)-\mathrm{H} 4 \ldots \mathrm{O} 3$ and $\mathrm{C}\left(\mathrm{sp}^{3}\right)-\mathrm{H} 16 \mathrm{~b} \ldots \pi$ hydrogen bonds and contribute $-4.95 \mathrm{kcal} \mathrm{mol}^{-1}$ towards stabilization. However, additional stabilization of -3.51 and $-1.93 \mathrm{kcal} \mathrm{mol}^{-1}$ was observed to be provided by motif $5(\mathrm{C} 15-\mathrm{H} 15 \mathrm{~b} . . \mathrm{O} 2)$ and $6(\mathrm{C} 14-\mathrm{H} 14 \mathrm{a} . . . \mathrm{C} 13)$ respectively.

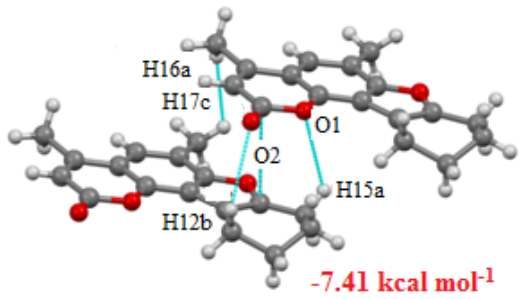

1

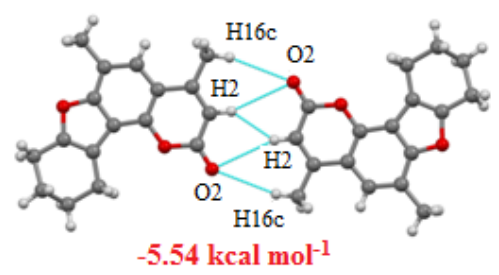

3
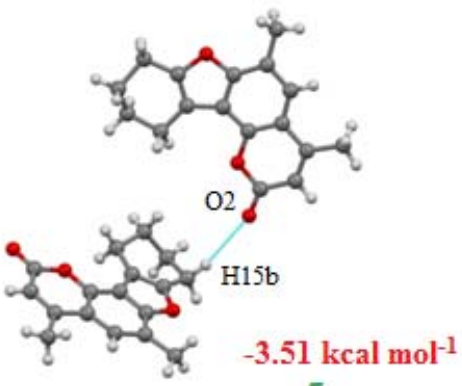

5

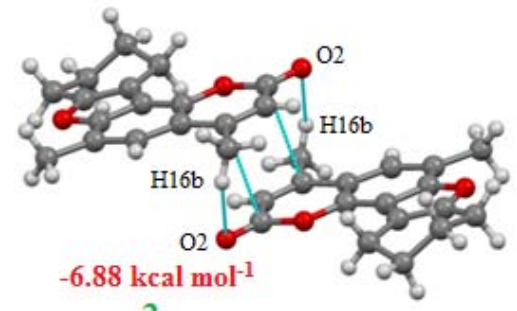

2

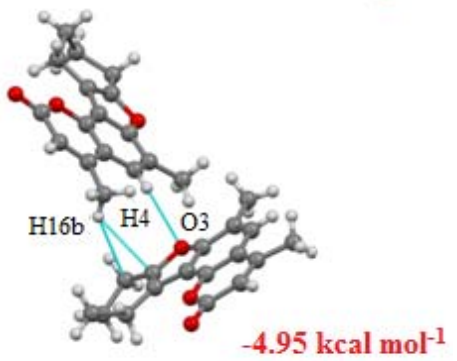

4

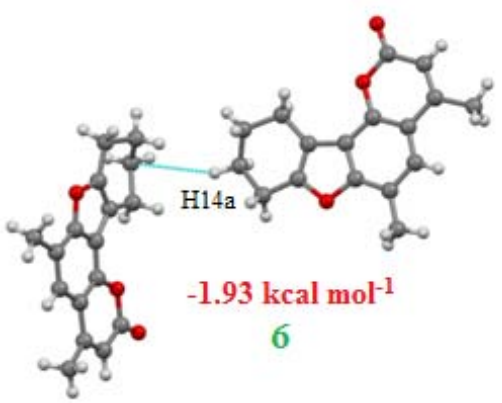

Figure 12. Molecular pairs (1-6) along with their interaction energies in C-6 


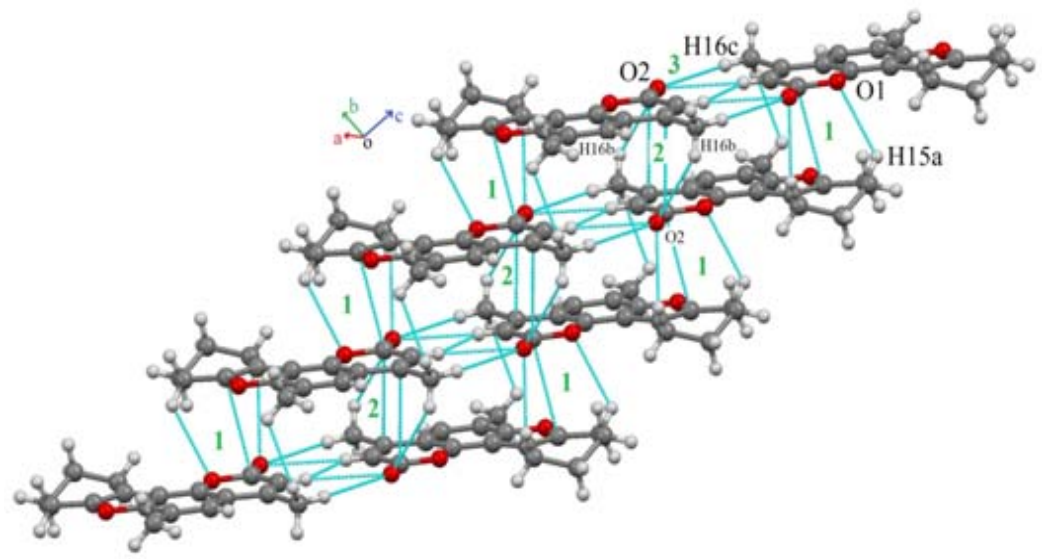

Figure 13. Packing of the molecules viewed down bc plane in C-6

3-Methylbutanoic acid (8S,9R)-8,9-dihydro-8-(1-hydroxy-1-methylethyl)-2-oxo-2Hfuro[2,3-h]-1-benzopyran-9-yl ester (C-7)

The different structural motifs (1-12) contributing towards the crystal packing are shown in Figure 14. The molecule crystallizes with two molecules in the asymmetric unit $[$ molecule $\mathrm{A}$ (carbon atom $=$ grey colour) and $\mathrm{B}$ (carbon atom $=$ violet colour)]. In this compound, there exists three types of molecular pairs $\mathrm{A}-\mathrm{A}, \mathrm{A}-\mathrm{B}$ and $\mathrm{B}-\mathrm{B}$ and energetically $\mathrm{A}-\mathrm{B}$ type molecular pairs are more stable than the $\mathrm{A}-\mathrm{A}$ and $\mathrm{B}-\mathrm{B}$ type. The two molecules in the asymmetric unit are connected with a strong O-H...O and weak $\mathrm{C}-\mathrm{H}$... O (involving $\mathrm{H}_{40}^{\prime}$ and $\mathrm{H}_{14} \mathrm{a}^{\prime}$ with bifurcated acceptor atom $\mathrm{O} 4$ ) hydrogen bonds and this pair is second most stabilized pair in the crystal, energy being $-9.85 \mathrm{Kcal} \mathrm{mol}^{-}$ ${ }^{1}$, the principal stabilization of around $50 \%$ corresponding to coulombic component. The most stabilized molecular pair in the crystal structure is stacked along the crystallographic $\mathrm{c}$ axis via $\pi \ldots \pi$ stacking along with weak $\mathrm{C}-\mathrm{H} \ldots \mathrm{O}$ hydrogen bonds and has a contribution of $-11.81 \mathrm{kcal} \mathrm{mol}^{-1}$ (motif 1) to the stabilization of the crystal packing. These stacks are then interconnected via motif 2,3,4,6 and 8 as depicted in Figure 15a. Molecular pair 5 (bifurcated C-H...O , I.E. $\left.=-4.97 \mathrm{kcal} \mathrm{mol}^{-1}\right)$ and $7(\mathrm{C} 3-$ H3...O6, I.E. $=-4.47 \mathrm{kcalmol}^{-1}$ ) propagates along the same direction forming chains. The two different chains formed by these motifs are interconnected via more stabilized motif 3 (Fig. 15c). Motif 3 involves the presence of $\mathrm{C} \ldots \pi(\mathrm{C} 19 \ldots \mathrm{Cg} 1)$ interaction along with $\mathrm{C}-\mathrm{H} . . . \mathrm{O}$ and $\mathrm{C}-\mathrm{H} \ldots \pi$. The combined nature of these interactions is mainly of dispersive nature. The packing in the crystal also displays the formation of molecular chain utilizing motif $6\left(-4.61 \mathrm{kcalmol}^{-1}\right)$ which consist of weak C-H...O and C-H... hydrogen bond. Motif $7\left(-4.47 \mathrm{kcal} \mathrm{mol}^{-1}\right)$ and $9(\mathrm{C}-\mathrm{H}$... O interaction involving $\mathrm{H} 13 \mathrm{~b}$ with $\mathrm{O} 2$ and $\mathrm{H} 14 \mathrm{c}$ with O6, I.E. $=-2.89 \mathrm{kcalmol}^{-1}$ ) interconnects the chains formed by motif 6 as shown in Figure 15b.

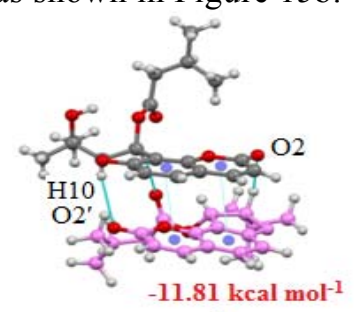

1

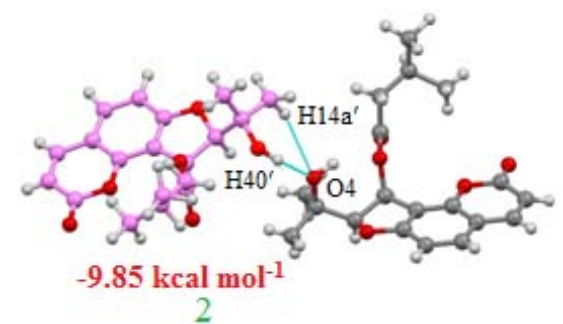

2 


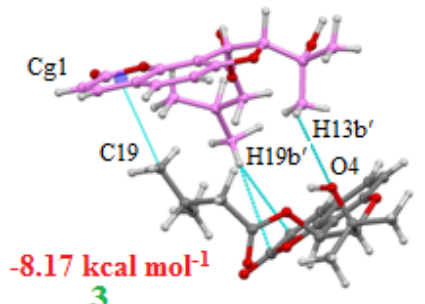

3
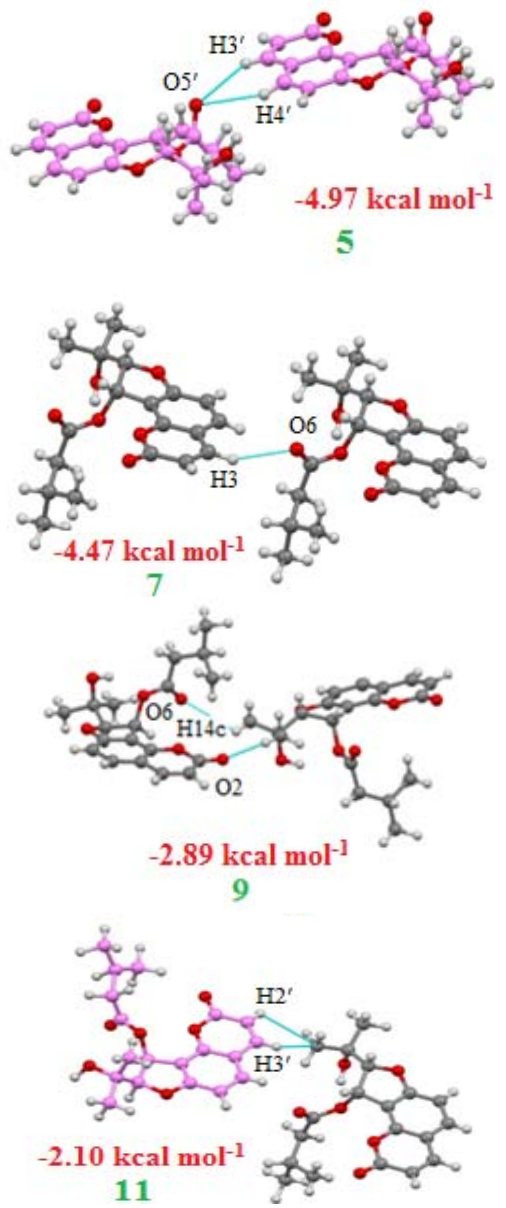
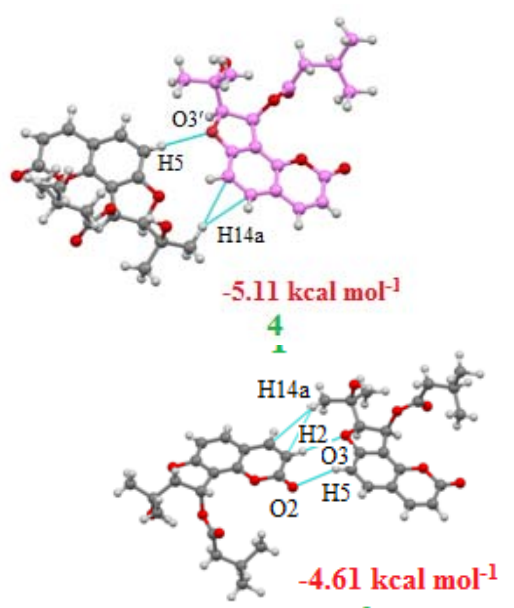

6

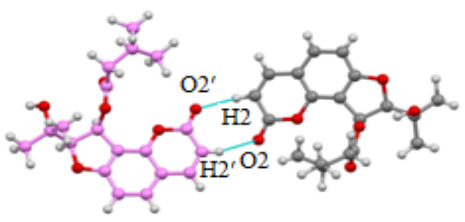

$-4.34 \mathrm{kcal} \mathrm{mol}^{-1}$

8

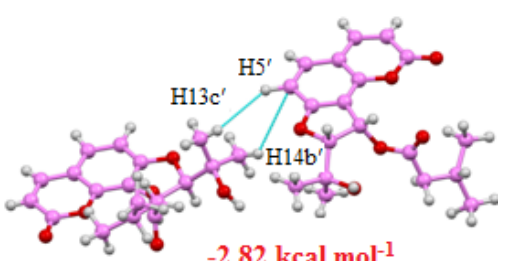

$-2.82 \mathrm{kcal} \mathrm{mol}^{-1}$

10

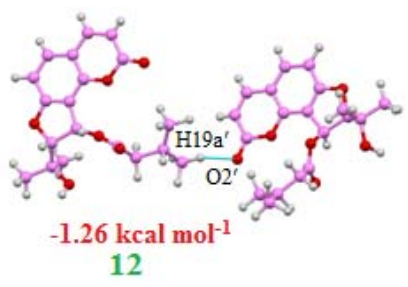

Figure 14. Molecular pairs (1-12) along with their interaction energies in C-7

A careful analysis of the different structural motifs obtained in these compounds leads to the following relevant observations:

1. The structural motifs providing maximum stabilization to the structure shows the presence of C-H...O hydrogen bonds or $\pi . . . \pi$ stacking interactions.

2. The interaction energies of the motifs involving $\pi \ldots \pi$ stacking interactions were observed to be in range -4.5 to $-11.5 \mathrm{kcal} \mathrm{mol}^{-1}$. These molecular pairs show significantly high dispersion component when compared to related molecular pairs showing the presence of intermolecular hydrogen bonds in the crystal structure. 
3. The energy of the molecular pairs interacting via $\mathrm{C}-\mathrm{H}$... O hydrogen bonds lies mostly in the range -2 to $-6 \mathrm{kcal} \mathrm{mol}^{-1}$ whereas those interacting via C-H... $\pi$ lies in the range- 1 to $-4 \mathrm{kcal} \mathrm{mol}^{-1}$.

4. The total interaction energy i.e lattice energy of the compounds lie in the range -20 to $33 \mathrm{kcal} \mathrm{mol}^{-1}$ and the main contribution to lattice energy comes from dispersion. The lattice energy is maximum in case of $\mathrm{C}-7$ containing two molecules in the asymmetric unit cell. The extra stability of C-7 arises mainly from its coulombic energy.
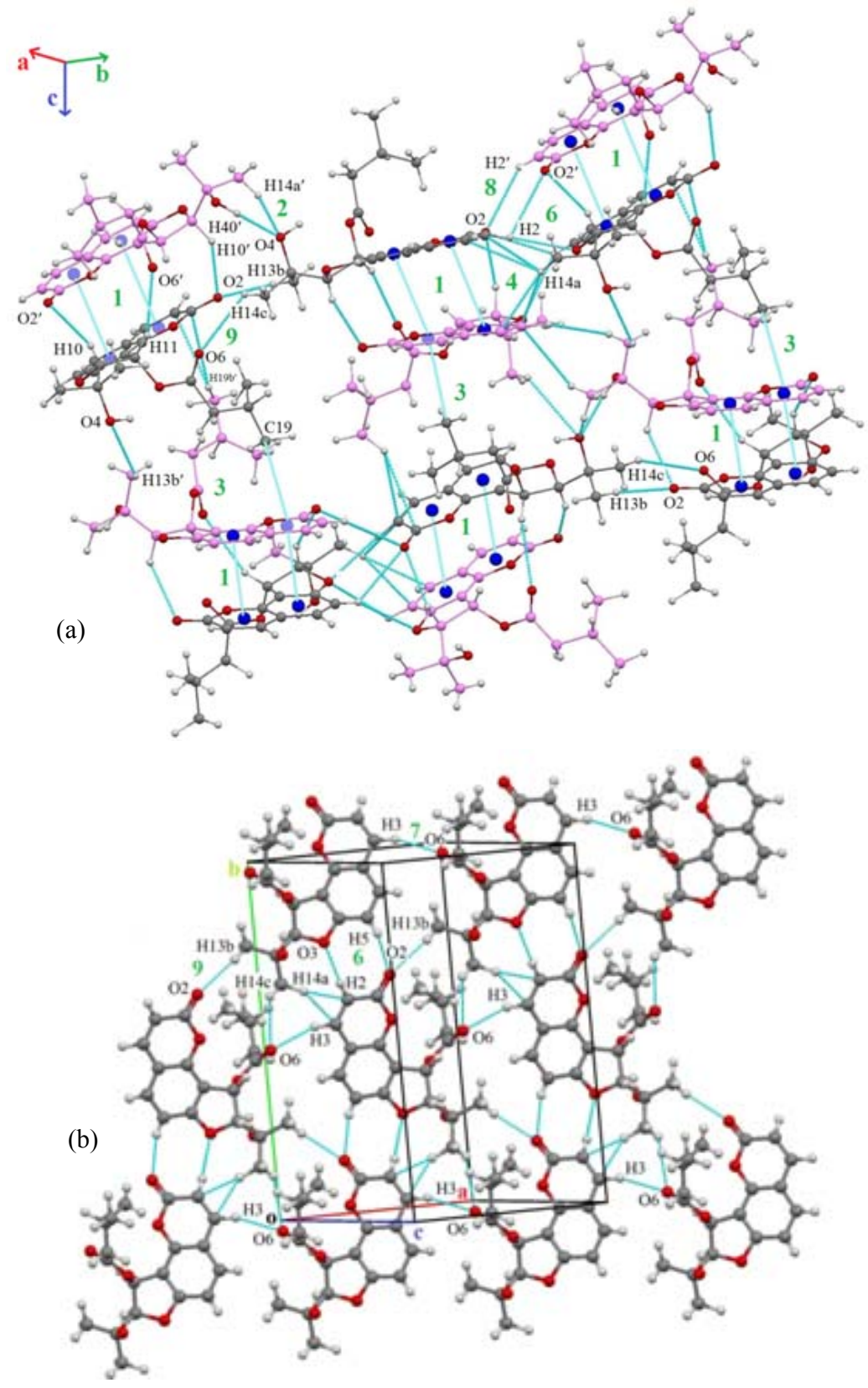


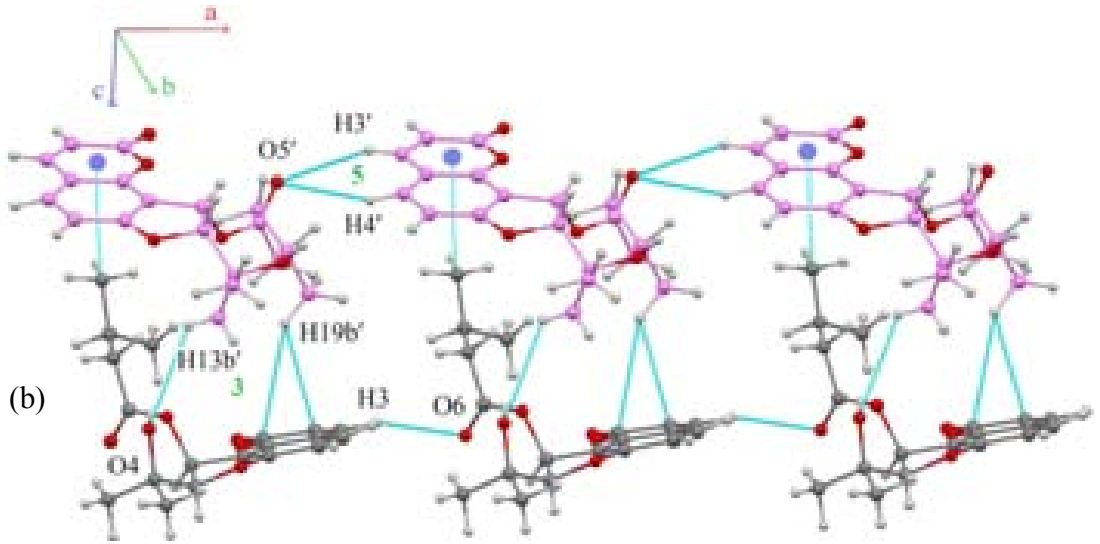

Figure 15. Packing of the molecules in C-7 viewed down (a) bc plane (b) ab plane and (c) ac plane

\section{Conclusion}

The present work provides a quantitative evaluation of energy of formation of various molecular pairs and their contribution towards stability of the packing. Analysis of geometry and energetics associated with the molecular pairs play an important role in the field of Crystal Structure Prediction. It is basically the interaction between the neighbouring molecules rather than the interaction between the atoms of the neighbouring molecules that influence the crystal structure and are vital in analyzing the cohesive energy of the crystal. Molecular pairs obtained after PIXEL calculation shows the presence of different structural motifs and their analysis suggests that considerable stabilization to the crystal packing is imparted by weak intermolecular interactions mainly $\pi \ldots \pi, \mathrm{C}-\mathrm{H} \ldots \mathrm{O}$ and $\mathrm{C}-\mathrm{H} \ldots \pi$.

\section{References}

1. Conforti F, Marrelli M, Menichini F, Bonesi M, Statti G, Provenzano E and Menichini F, Curr Drug Ther., 2009, 4(1), 38-58; DOI:10.2174/157488509787081886

2. Borges F, Roleira F, Milhazes N, Santana L and Uriarte E, Curr Med Chem., 2005, 12, 887-916.

3. Gambari R, Lampronti I, Bianchi N, Zuccato C, Viola G, Vedaldi D and Dall Acqua F, Top Heterocycl Chem., 2007, 9, 265.

4. $\quad$ Bordin F, Dall Acqua F and Guiotto A, Pharmacol Ther., 1991, 52(3), 331-369; DOI:10.1016/0163-7258(91)90031-G

5. Gould J W, Mercurio M G and Elmets C A, J Am Acad Dermatol., 1995, 33, 571--573.

6. Stern R S, Lancet, 1994, 344(8937), 1644-1646; DOI:10.1016/S0140-6736(94)90442-1

7. Hickey B M, Almarsson Ö and Peterson M L, Cryst Eng Comm., 2012, 14, 2349; DOI:10.1039/C2CE90019J

8. Desiraju G R, Angew Chem Int Ed., 2011, 50(1), 52-59; DOI:10.1002/anie.201002960

9. Desiraju G R, Chem Commun., 2005, 2995-3001; DOI:10.1039/B504372G

10. Bosch E, Cryst Growth Des., 2010, 10(8), 3808-3813; DOI:10.1021/cg100707y

11. Panini P and Chopra D, Cryst Eng Comm., 2012, 14, 1972-1989; DOI:10.1039/ C2CE06254B

12. Panini P and Chopra D, Cryst Eng Comm., 2013, 15, 3711-3733; DOI:10.1039/ C3CE40111A

13. Nishio M, Phys Chem Chem Phys., 2011, 13, 13873-13900; DOI:10.1039/C1CP20404A 
14. Martinez C R and Iverson B L, Chem Sci., 2012, 3, 2191-2201; DOI:10.1039/C2SC$20045 \mathrm{G}$

15. Shukla R, Mohan T P, Vishalakshi B and Chopra D, Cryst Eng Comm., 2014, 16, 1702-1713; DOI:10.1039/C3CE42286K

16. Gavezzotti A, New J Chem., 2011, 35, 1360-1368; DOI:10.1039/C0NJ00982B

17. Maschio L, Civalleri B, Ugliengo P and Gavezzotti A, Phys Chem A, 2011, 115(41), 11179-11186; DOI:10.1021/jp203132k

18. Bravic G, Bideau J P and Desvergne J P, Cryst Struct Commun., 1980, 9, 705.

19. Gupta V K, Rajnikant, Goswami K N, Mazumdar S K, Gupta B D and Banerjee S K, Cryst Res Technol., 1993, 28(2), 187-191; DOI:10.1002/crat.2170280211

20. De Oliveira P E S, Conserva L M, De Simone C A, Pereira M A, Malta V R S and Imbroisi D O, Acta Crystallogr Sect C: Cryst Struct Commun., 2004, 60(12), o900o902; DOI:10.1107/S0108270104023777

21. Magotra D K, Gupta V K, Rajnikant, Goswami K N and Gupta B D, Acta Crystallogr Sect C: Cryst Struct Commun., 1995, 51(12), 2637-2639;

DOI:10.1107/S0108270195008353

22. Mosti L, Schenone P, Menozzi G, Sancassan F, Baccichetti F, Benetollo F and Bombieri G, J Heterocycl Chem., 1988, 25(2), 407-413; DOI:10.1002/jhet.5570250211

23. Caffieri S, Miolo G, Dall Acqua F, Benetollo F and Bombieri G, Photochem Photobiol., 2000, 72(1), 23-27; DOI:10.1562/0031-8655(2000)0720023PODTTI2.0.CO2

24. Singh A, Gupta V K, Rajnikant and Goswami K N, Mol Mat., 1995, 5, 289. 